Prostaglandin E-2-EP2-NF-kappa B signaling in macrophages as a potential therapeutic target for intracranial aneurysms

\title{
Aoki, Tomohiro
}

2017-02-07

Aoki , T, Frösen , J , Fukuda , M , Bando , K, Shioi , G, Tsuji , K, Ollikainen , E , Nozaki , K , Laakkonen , J \& Narumiya , S 2017 , ' Prostaglandin E-2-EP2-NF-kappa B signaling in macrophages as a potential therapeutic target for intracranial aneurysms ' , Science signaling , vol. 10 , no. 465 , 6037 . https://doi.org/10.1126/scisignal.aah6037

http://hdl.handle.net/10138/233572

https://doi.org/10.1126/scisignal.aah6037

publishedVersion

Downloaded from Helda, University of Helsinki institutional repository.

This is an electronic reprint of the original article.

This reprint may differ from the original in pagination and typographic detail.

Please cite the original version. 


\title{
Prostaglandin $\mathrm{E}_{2}-\mathrm{EP} 2-\mathrm{NF}-\mathrm{\kappa B}$ signaling in macrophages as a potential therapeutic target for intracranial aneurysms
}

2017 (c) The Authors, some rights reserved: exclusive licensee American Association for the Advancement of Science.

\author{
Tomohiro Aoki, ${ }^{1,2}$ Juhana Frösen, ${ }^{3,4,5}$ Miyuki Fukuda, ${ }^{1}$ Kana Bando, $^{6,7}$ Go Shioi, ${ }^{7}$ Keiichi Tsuji, $^{8}$ \\ Eliisa Ollikainen, ${ }^{3}$ Kazuhiko Nozaki, ${ }^{8}$ Johanna Laakkonen, ${ }^{9}$ Shuh Narumiya ${ }^{1,2 *}$
}

Intracranial aneurysms are common but are generally untreated, and their rupture can lead to subarachnoid hemorrhage. Because of the poor prognosis associated with subarachnoid hemorrhage, preventing the progression of intracranial aneurysms is critically important. Intracranial aneurysms are caused by chronic inflammation of the arterial wall due to macrophage infiltration triggered by monocyte chemoattractant protein-1 (MCP-1), macrophage activation mediated by the transcription factor nuclear factor $\kappa B(\mathrm{NF}-\kappa \mathrm{B})$, and inflammatory signaling involving prostaglandin $E_{2}\left(P_{G}\right)$ and prostaglandin $E$ receptor subtype 2 (EP2). We correlated EP2 and cyclooxygenase-2 (COX-2) with macrophage infiltration in human intracranial aneurysm lesions. Monitoring the spatiotemporal pattern

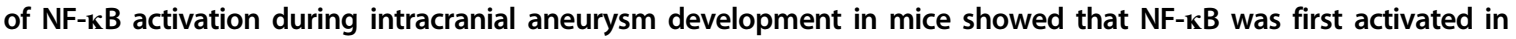
macrophages in the adventitia and in endothelial cells and, subsequently, in the entire arterial wall. Mice with a

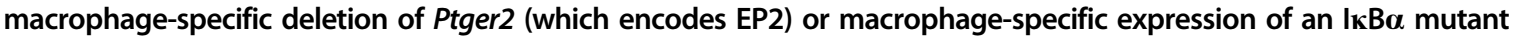

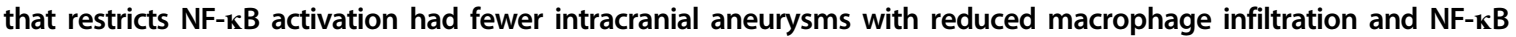
activation. In cultured cells, EP2 signaling cooperated with tumor necrosis factor- $\alpha$ (TNF- $\alpha$ ) to activate NF- $\mathrm{KB}$ and synergistically induce the expression of proinflammatory genes, including Ptgs2 (encoding COX-2). EP2 signaling also stabilized $\mathrm{CCl} 2$ (encoding MCP-1) by activating the RNA-stabilizing protein HuR. Rats administered an EP2 antagonist had reduced macrophage infiltration and intracranial aneurysm formation and progression. This signaling pathway in macrophages thus facilitates intracranial aneurysm development by amplifying inflammation in intracranial arteries. These results indicate that EP2 antagonists may therefore be a therapeutic alternative to surgery.

\section{INTRODUCTION}

Subarachnoid hemorrhage is the most severe form of cerebrovascular disease and a fatal illness; up to $50 \%$ of patients die within 30 days after the onset and $30 \%$ suffer from moderate to severe morbidity (1). The main cause of subarachnoid hemorrhage is rupture of a preexisting intracranial aneurysm, which affects 1 to $5 \%$ of the general public $(2,3)$. Given the high prevalence and susceptibility to subarachnoid hemorrhage, treatment of intracranial aneurysms to prevent rupture is clinically important. Because of the lack of medical therapy for intracranial aneurysms, only patients with "rupture-prone" intracranial aneurysms, such as those that are more than $7 \mathrm{~mm}$ in diameter or those that are located at specific bifurcation sites, are surgically treated. Surgery carries the unavoidable risk of complications $(4,5)$, and the number of untreated patients remains very high. Neither of these outcomes are desirable; less than half of intracranial aneurysms [3050 lesions among 6697 unruptured intracranial aneurysms (45.5\%)] were surgically treated in a large Japanese cohort study

\footnotetext{
${ }^{1}$ Center for Innovation in Immunoregulation Technology and Therapeutics, Kyoto University Graduate School of Medicine, Kyoto 606-8501, Japan. ${ }^{2}$ Core Research for Evolutional Science and Technology, Medical Innovation Center, Kyoto University Graduate School of Medicine, Kyoto 606-8507, Japan. ${ }^{3}$ Neurosurgery Research Group, Biomedicum Helsinki, Helsinki 00029 HUS, Finland. ${ }^{4}$ Hemorrhagic Brain Pathology Research Group, NeuroCenter, Kuopio University Hospital, Kuopio 70029 KYS, Finland. ${ }^{5}$ Department of Neurosurgery, NeuroCenter, Kuopio University Hospital, Kuopio 70029 KYS, Finland. ${ }^{6}$ Animal Resource Development Unit, RIKEN Center for Life Science Technologies, Hyogo 650-0047, Japan. ${ }^{7}$ Genetic Engineering Team, RIKEN Center for Life Science Technologies, Hyogo 650-0047, Japan. ${ }^{8}$ Department of Neurosurgery, Shiga University of Medical Science, Shiga 520-2192, Japan. ${ }^{9}$ Department of Molecular Medicine, A.I. Virtanen Institute, University of Eastern Finland, Kuopio 70211, Finland.

*Corresponding author. Email: snaru@mfour.med.kyoto-u.ac.jp
}

(6). A safe and noninvasive medical therapy that prevents the progression and rupture of intracranial aneurysms is therefore urgently needed, and to accomplish this goal, the mechanism responsible for formation and progression of intracranial aneurysms must be elucidated.

Intracranial aneurysms are lesions that are histologically characterized by chronic inflammation and degenerative changes in the arterial walls (7). These characteristics can be recapitulated in an animal model, in which intracranial aneurysms are induced by renal hypertension and unilateral ligation of the carotid artery $(8,9)$. Because intracranial aneurysms occur at intracranial artery bifurcation sites both clinically and in animal models, and computational simulation of fluid hemodynamics shows a high wall shear stress at these sites, it is thought that shear stress is a trigger for intracranial aneurysm formation (10-12). High wall shear stress is thought to evoke an inflammatory response in endothelial cells, which then presumably spreads to the whole arterial wall after macrophage recruitment and secretion of proinflammatory factors $(7,13)$. Human intracranial aneurysms with high wall shear stress have more inflammatory cells in their walls (14). The transcription factor nuclear factor $\kappa \mathrm{B}(\mathrm{NF}-\kappa \mathrm{B})$ appears to play a crucial role in these processes $(7,13,15)$. The loss of the NF- $\kappa \mathrm{B}$ p50 subunit or pharmacological inhibition of its transcriptional activity by intrathecal administration of decoy oligonucleotides suppresses intracranial aneurysm formation and progression in rodent models and suppresses inflammatory responses in the lesion, verifying that NF- $\kappa \mathrm{B}$ mediates inflammation in the arterial wall and is critical for the formation and progression of intracranial aneurysms $(7,15,16) . \mathrm{NF}-\kappa \mathrm{B}$ and its downstream effectors are therefore potential therapeutic targets for the treatment of intracranial aneurysms. However, because NF- $\mathrm{KB}$ signaling is also important in various physiological processes, $\mathrm{NF}-\mathrm{\kappa B}$-activating pathways that are restricted to 
the inflammatory setting within intracranial aneurysms would be an ideal target.

Prostaglandins (PGs), including $\mathrm{PGD}_{2}, \mathrm{PGE}_{2}, \mathrm{PGF}_{2 \alpha}$, and $\mathrm{PGI}_{2}$, and thromboxane $\mathrm{A}_{2}$ are arachidonic acid metabolites formed by the sequential enzymatic action of cyclooxygenase (COX) and respective synthases and exert their actions by acting on their cognate $G$ protein (heterotrimeric guanine nucleotide-binding protein)-coupled receptors (17). Using mice deficient in individual PG receptors, we have shown that PGE receptor subtype 2 (EP2) signaling is involved in $\mathrm{NF}-\kappa \mathrm{B}$ activation and inflammation in the wall of intracranial aneurysms $(9,18)$. An inducible isoform of COX, COX-2, membrane-bound PGE synthase (mPGES-1), and EP2 all show increased abundance in arterial walls during the formation of an intracranial aneurysm; furthermore, the loss of EP2 suppresses NF- $\kappa \mathrm{B}$ activation, inflammation, and intracranial aneurysm formation (18). However, the mechanism by which EP2 signaling is linked to NF- $\kappa$ B activation and the types of cells in which this pathway operates remain obscure. Moreover, it remains unclear whether pharmacological manipulation of this pathway would have the desired therapeutic effect. Here, we found that EP2 signaling directly activates NF- $\kappa \mathrm{B}$ p50/p65, which cooperates with tumor necrosis factor $-\alpha$ (TNF- $\alpha$ )-activated NF- $\kappa$ B and synergistically induces the expression of various inflammation-related genes including Ptgs2 (which encodes COX-2). In addition, we show that deletion of Ptger2 (which encodes EP2) and suppression of NF- $\mathrm{KB}$ specifically in macrophages inhibited macrophage infiltration and prevented intracranial aneurysm formation. We further found that administration of an EP2 antagonist prevents not only the formation but also the progression of intracranial aneurysms. Because EP2 is abundant in macrophages in human intracranial aneurysm lesions, this suggests that EP2 antagonism is a promising therapeutic strategy to prevent the progression of intracranial aneurysms.

\section{RESULTS}

COX-2 and EP2 are associated with macrophage infiltration in the wall of intracranial aneurysms in humans

Previous studies in animal models have shown that NF- $\kappa \mathrm{B}$ signaling in macrophages plays a crucial role in the inflammation underlying intracranial aneurysm pathogenesis and implicate EP2 in the inflammatory processes $(13,15,18,19)$. We first aimed to clarify the clinical relevance of these findings. We used immunostaining to assess EP2 and COX-2 abundance in human intracranial aneurysm lesions (Fig. 1A) in surgical samples obtained from 36 patients (20). We correlated the abundance of these proteins with other parameters of inflammation, particularly macrophage infiltration into the walls of the intracranial aneurysms. We performed immunostaining for CD68 to detect macrophages and for COX-2 and EP2 in adjacent tissue sections from aneurysm lesions $(21,22)$. Both EP2 and COX-2 were detected in all layers of the intracranial aneurysm wall, including the endothelial layer and the adventitia. In addition, EP2 and COX-2 immunostaining appeared as numerous puncta in the inner half of the media, where smooth muscle cells were lost because of tissue degeneration (Fig. 1A, left and middle); this smooth muscle loss is characteristic of ruptured intracranial aneurysms (22). CD68-positive cells were also scattered in a similar pattern in the media and the adventitia, suggesting macrophage infiltration in these regions (Fig. 1A, right). When the density of EP2 and COX-2 signals in the whole arterial wall was scored in each sample (fig. S1), and compared to the number of CD68-positive macrophages in adjacent sections, the EP2 and COX-2 signal scores in the
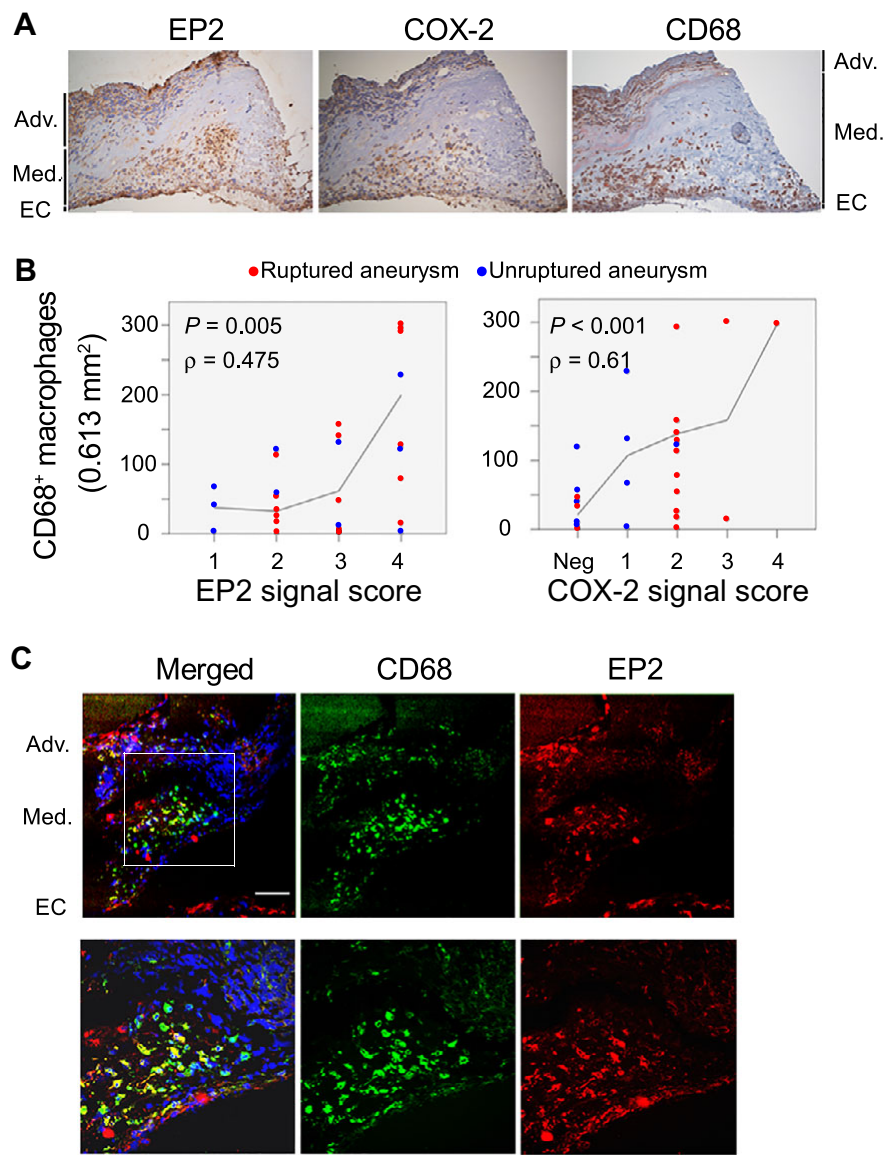

Fig. 1. EP2 and COX-2 distribution in human intracranial aneurysms. (A) Adjacent tissue sections were prepared from unruptured or ruptured human intracranial aneurysm samples and stained for EP2 (left), COX-2 (middle), and CD68 (right, with Oil Red $\mathrm{O}$ background staining). Representative image from ruptured intracranial aneurysm is shown. Adv., adventitia; Med., media; EC, endothelial cell layer. Scale bar, $100 \mu \mathrm{m}$. (B) The intensity of EP2 and COX-2 immunostaining was scored according to the criteria described in fig. S1, and the correlation of signal intensity with the number of CD68-positive macrophages in the lesion was examined using the Spearman rank correlation test. Red and blue circles indicate data from ruptured and unruptured aneurysms, respectively. $n=36$ human intracranial aneurysm specimens. (C) Colocalization of EP2 signals (red) with those of CD68 (green) in the adventitia and media. Merged images with nuclear 4',6-diamidino-2-phenylindole (DAPI) staining are also shown in the left panel. Images with higher magnification corresponding to the square in the upper panel are shown in the lower panels. $n=14$ human intracranial aneurysm samples. Scale bar, $100 \mu \mathrm{m}$.

lesion correlated both positively and significantly with the number of infiltrated macrophages (Fig. 1B). Furthermore, double immunohistochemistry showed that the EP2 signals largely colocalized with those for CD68 in the adventitia and media (Fig. 1C). In addition, EP2 signals colocalized with the endothelial cell marker CD31 in the endothelium and with the smooth muscle cell marker $\alpha$-smooth muscle actin in the media where degenerative change was limited (fig. S2, A to F).

\section{NF- $\kappa B$ activation is spatiotemporally regulated in intracranial aneurysm lesions}

Our finding that EP2 was present in macrophages, endothelial cells, and smooth muscle cells in human intracranial aneurysm tissue samples raised the question about the time course of these changes in EP2 
abundance during intracranial aneurysm development. Given the crucial role of NF- $\kappa \mathrm{B}$ as a key mediator of inflammation in intracranial aneurysm lesions $(7,15)$ and our previous finding that EP2 signaling activates NF- $\kappa \mathrm{B}$ in primary cultured endothelial cells in vitro (18), we wanted to follow the course of inflammation during intracranial aneurysm development by monitoring NF- $\kappa \mathrm{B}$ activation in the arterial walls of intact animals. To this end, we made a construct encoding a derivative of red fluorescent protein (RFP), Timer-1 (23), with consensus $\mathrm{NF}-\kappa \mathrm{B}$ binding sites in its promoter region (fig. S3A). Expression of this Timer-1 construct yielded potent RFP signals in response to TNF- $\alpha$ in human embryonic kidney (HEK) 293 cells (fig. S3B). We then generated a transgenic mouse line expressing this construct and subjected the mice to NF-KB-activating stimuli. Intraperitoneal injection of lipopolysaccharide (LPS) induced Timer-1 protein expression in the liver of these mice, and dextran sodium sulfate (DSS)-induced colitis induced Timer-1 expression in the colon (fig. S3, C and D). We then induced intracranial aneurysm formation in this line of NF- $\mathrm{KB}$ reporter mice. Before intracranial aneurysm induction, Timer-1 signals were not detected at the bifurcation sites of intracranial arteries (Fig. $2 \mathrm{~A}$ ). NF- $\kappa \mathrm{B}$ activation, as assessed by Timer- 1 expression, was first detected on the luminal surface and the adventitia of the arterial wall 2 weeks after intracranial aneurysm induction and spread to neighboring regions with increasing intensity by 4 weeks. At this time point, Timer-1 signals in the adventitia colocalized with those for the macrophage marker F4/80 and those at the luminal surface with the endothelial marker CD31 (fig. S4), suggesting that NF- $\kappa \mathrm{B}$ was activated in macrophages infiltrating into the adventitia and in endothelial cells at this stage of intracranial aneurysm formation. At 8 weeks after induction, NF- $\kappa \mathrm{B}$ activation was observed in the whole arterial wall (Fig. 2A), and coimmunofluorescence analysis indicated that it also occurred in smooth muscle cells in the media, macrophages in the adventitia, and endothelial cells (fig. S4). Once triggered, NF- $\mathrm{KB}$ activation, as monitored by Timer- 1 expression, was sustained in the intracranial aneurysm lesions until at least 32 weeks (Fig. 2A). However, because intracranial aneurysm progression is limited in this mouse model and the smooth muscle layer is almost intact during the experimental period $(8,24)$, we did not monitor NF- $\mathrm{BB}$ activation in the intracranial aneurysm walls up to the stage of excessive degeneration of the media and loss of medial smooth muscle cells, as was detected in human intracranial aneurysms (Fig. 1).

\section{$P_{2}$-EP2 signaling in macrophages sustains inflammation, leading to intracranial aneurysm formation}

EP2 signaling activates NF- $\mathrm{KB}$ in primary cultured endothelial cells in vitro (18). To determine whether EP2 signaling also activated NF- $\kappa B$ activation in vivo in intracranial aneurysm lesions, we used Ptger2deficient mice (25) crossed with NF- $\mathrm{\kappa B}$ reporter mice. Ptger 2 deficiency suppressed NF- $\kappa \mathrm{B}$ activation in intracranial arteries 4 and 8 weeks after intracranial aneurysm induction in a gene dosage-dependent manner (Fig. 2B). These data implied that EP2 signaling was involved in NF- $\kappa \mathrm{B}$ activation in endothelial cells and macrophages at the beginning of intracranial aneurysm formation as well as in the entire arterial wall at later stages of intracranial aneurysm formation.

To determine the cell type that was important for EP2-induced $\mathrm{NF}-\kappa \mathrm{B}$ activation and in intracranial aneurysm formation, we generated Ptger $2^{\text {flox/flox }}$ mice. Using the loxP-Cre recombinase system, we deleted the first exon of Ptger2, which encodes the first to the middle of the sixth transmembrane domains of the EP2 protein. The mice produced by this approach had a similar genotype as the Ptger2-deficient mice we generated previously (fig. S5, A to C) (25). Cre-dependent deletion of the first exon of Ptger2 was confirmed by direct sequencing of genomic DNA from the kidneys of Ptger $2^{\text {flox/flox }}$-EIIa-Cre mouse. Macrophage- or endothelial cell-specific deletion of Ptger2 was then achieved by crossing Ptger $2^{\text {flox/flox }}$ mice with $L y z 2$-Cre mice (Ptger $2^{\mathrm{f} / \mathrm{f}}$ Lyz2Cre mice), which express Cre specifically in macrophages under the control of the Lyz2 (which encodes lysozyme 2) promoter, or Cdh5-Cre mice (Ptger ${ }^{\mathrm{f} / \mathrm{f}} \mathrm{Cdh} 5 \mathrm{Cre}$ mice), which express Cre specifically in endothelial cells under the control of $C d h 5$ (which encodes VE-cadherin) promoter, respectively. We first assessed NF- $\kappa \mathrm{B}$ activation by performing immunohistochemistry for the NF- $\kappa \mathrm{B}$ p 65 subunit phosphorylated at $\operatorname{Ser}^{536}$ in these two lines of transgenic mice after intracranial aneurysm induction. The macrophage-specific deletion of Ptger 2 prevented NF- $\kappa \mathrm{B}$ activation in endothelial cells 2 weeks after surgical manipulation, an early stage of intracranial aneurysm formation (Fig. 2C), and almost abolished NF- $\kappa \mathrm{B}$ activation in whole intracranial arterial walls at the later stage (20 weeks after surgical manipulation) (Fig. 2D). Notably, macrophage-specific deletion of Ptger 2 suppressed the abundance of proteins regulated by NF- $\mathrm{KB}$ activation, namely, monocyte chemoattractant protein-1 (MCP-1) and COX-2, in the adventitia of intracranial aneurysm lesions where macrophages accumulated (fig. S6), suggesting that regulation of NF- $\kappa \mathrm{B}$ activation by EP2 signaling occurred in macrophages in vivo. In contrast, Ptger 2 deletion specifically in endothelial cells (Ptger $2^{\mathrm{f} / \mathrm{f}} \mathrm{Cdh} 5 \mathrm{Cre}$ mice) abolished the signals in the endothelium but did not affect NF- $\kappa \mathrm{B}$ activation in the adventitia at 20 weeks (Fig. 2D).

Because these data suggested that EP2 signaling in endothelial cells and macrophages contributes to the inflammatory responses in these cell types at the initial stages and that EP2 signaling in macrophages may have sustained effects on inflammation in arterial walls, we next examined the contribution of the signaling in each cell type to intracranial aneurysm formation. Because the mouse model of intracranial aneurysm shows limited lesion progression and does not induce outward bulging of arterial walls, which is typically observed at the advanced stage (8), we evaluated intracranial aneurysm formation by examining breakage of the lamina elastica and also assessed macrophage infiltration using immunohistochemistry for F4/80-positive cells. Twenty weeks after surgical manipulation, Ptger $2^{\mathrm{f} / \mathrm{f}}$ Lyz2Cre mice, but not $\operatorname{Ptger} 2^{\mathrm{f} / \mathrm{f}} \mathrm{Cdh}$ 5Cre mice, had significantly less intracranial aneurysm formation (Fig. 2E), which was accompanied by suppression of $\mathrm{NF}-\kappa \mathrm{B}$ activation and macrophage recruitment to intracranial aneurysm lesions (Fig. 2, D and F). Deficiency of Ptger2 specifically in endothelial cells did not affect intracranial aneurysm formation (Fig. 2E) or macrophage infiltration of the lesions (Fig. 2F). These data suggest that EP2-mediated NF- $\mathrm{B}$ activation in macrophages, but not in endothelial cells, specifically contributes to intracranial aneurysm formation in vivo.

\section{NF- $\kappa$ B activation in macrophages is critical for intracranial aneurysm formation}

To corroborate the role of macrophage signaling in intracranial aneurysm inflammation and to examine whether NF- $\kappa \mathrm{B}$ activation specifically in macrophages is critical to intracranial aneurysm formation, we established a mouse line in which a mutated form of IкB $\alpha$ (S32A/ $\mathrm{S} 36 \mathrm{~A}-\mathrm{I} \kappa \mathrm{B} \alpha$ ) was expressed under the control of Cre recombinase. Expression of this IKB mutant protein could easily be monitored because of its fusion with mCherry (Fig. 3A and fig. S7, A to C). S32A/ $\mathrm{S} 36 \mathrm{~A}-\mathrm{I} \kappa \mathrm{B} \alpha$ is a superrepressor of NF- $\kappa \mathrm{B}(26)$ because it is resistant to phosphorylation-dependent degradation, thereby interfering with the nuclear translocation of NF- $\kappa \mathrm{B}$. The Cre-dependent expression of the 
$\mathrm{I} \kappa \mathrm{B}$ mutant, its resistance to degradation under NF- $\mathrm{KB}$-activating conditions, and its inhibitory effect on NF- $\kappa \mathrm{B}$ activation were confirmed in vitro either in HEK293 cells or in a stable NF- $\kappa$ B reporter HEK293 cell line [a cell line that expresses green fluorescent protein (GFP) under the transcriptional control of NF- $\kappa \mathrm{B}$ ] stimulated with TNF- $\alpha$ (fig. S8, A to C). The Cre-dependent expression of the IкB mutant was further confirmed by transfecting a Cre-expressing plasmid into primary intraperitoneal macrophages isolated from trans-

A

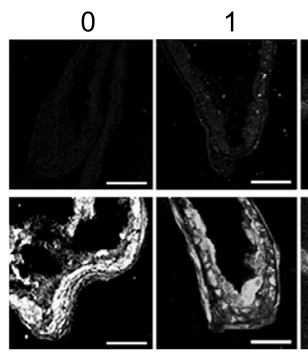

8

C

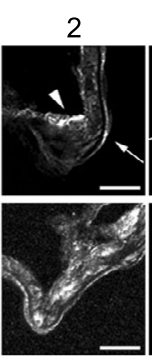

27
B

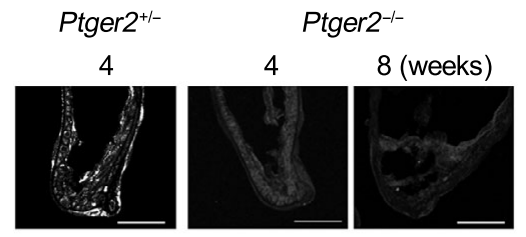

$\mathrm{NF}-\kappa \mathrm{B}$ reporter mouse
D

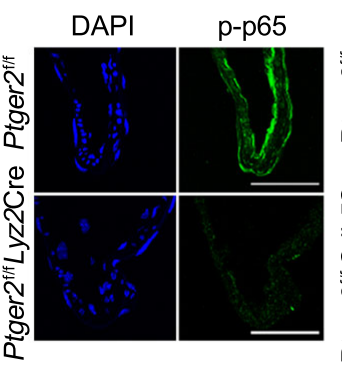

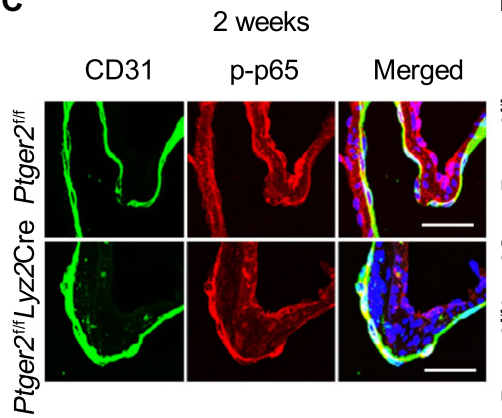

20 weeks

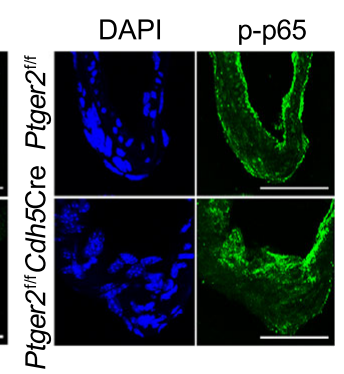

E

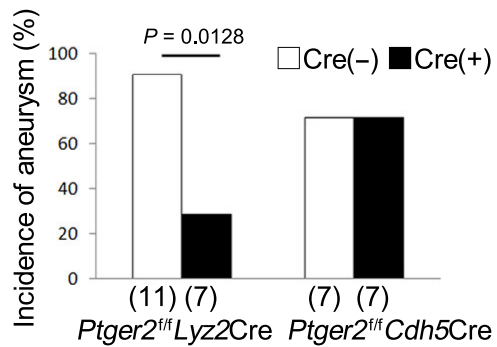

genic mice or by injecting a Cre-expressing plasmid into the femoral muscle of this mouse line (fig. S8, D and E). We crossed these I $\mathrm{B}$ mutant mice with either Lyz2-Cre mice or Cdh5-Cre mice. IкB mutantLyz2Cre mice and IкB mutant-Cdh5Cre mice (figs. S9, A and B, and

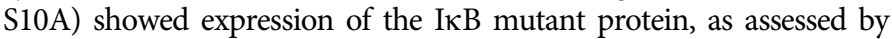
mCherry expression, specifically in F4/80-positive macrophages (fig. S9, A and C) and CD31-positive endothelial cells (fig. S10, B and $\mathrm{C})$, respectively. When intracranial aneurysms were induced in these mice, the incidence of intracranial aneurysms in the IкB mutant-Cdh5Cre mice was comparable to that in their littermates, whereas the I $\kappa$ B mutant-Lyz2Cre mice had a significantly lower incidence of intracranial aneurysms and also demonstrated reduced macrophage infiltration compared to their littermates (Fig. $3 \mathrm{~A})$ in the absence of changes in systemic blood pressure (fig. S11, A and B). These data are consistent with the effect of cell typespecific deletion of Ptger 2 on the sustained activation of NF- $\kappa \mathrm{B}$, as assessed by Timer- 1 expression, in the whole arterial wall (Fig. 2, C and D). As was observed in the Ptger $2^{\mathrm{f} / \mathrm{f}}$ Lyz2Cre mice (Fig. 2D), NF- $\kappa \mathrm{B}$ activation, as assessed by immunostaining for phosphorylated p65, was suppressed in the whole arterial wall in the I $\kappa \mathrm{B}$ mutant-Lyz2Cre mice compared to their littermates (Fig. 3B). Quantitative realtime polymerase chain reaction (RT-PCR) analysis revealed that intracranial aneurysm induction increased the expression of $\mathrm{Ccl} 2$ and Ptgs2, which encode two NF- $\mathrm{BB}$-induced proinflammatory proteins that are involved in intracranial aneurysm formation $(18,19,27)$. The increase in the expression of these two proinflammatory factors was ablated in macrophages expressing the I $\mathrm{B}$ mutant (Fig. $3 \mathrm{C}$ ). These findings were consistent with the presence of MCP-1 in macrophages infiltrating the intracranial aneurysm lesions, which was abolished in I $\kappa$ B mutant-Lyz2Cre mice (Fig. $3 \mathrm{D})$. These results indicate that NF- $\kappa \mathrm{B}$ activation in macrophages promotes inflammation in the whole arterial wall and critically contributes to intracranial aneurysm formation, at least partly, by forming a self-recruiting loop consisting of macrophages secreting their own chemoattractant, MCP-1.

aneurysm induction. (A) Time course of NF- $K$ B activation in intion in intracranial aneurysm walls and intracra pression. Arrows and arrowheads indicate signals in endothelial cells and macrophages, respectively. Scale bars, $20 \mu \mathrm{m}$. Representative images from three slices per mouse (five mice for 0, 1, 2, 3, 4, 8, and 20 weeks; three mice for

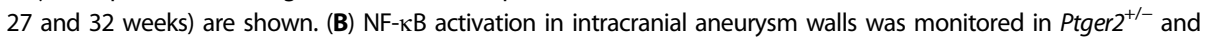
Ptger $^{-1-}$ NF-אB reporter mice 4 or 8 weeks after intracranial aneurysm induction. Scale bars, $20 \mu \mathrm{m}$. Representative images from mice $(n=5)$ from each genotype and each time point from three independent experiments are shown. (C and D) NF- $\kappa B$ activation examined by staining for phosphorylated p65 (Ser ${ }^{536}$; p-p65) in intracranial aneurysm lesions in Ptger2 ${ }^{\mathrm{f} / f}$ Lyz2Cre mice at an early stage (2 weeks) (C) and in Ptger2 ${ }^{\mathrm{f} / \mathrm{f}}$ Lyz2Cre and Ptger2 ${ }^{\mathrm{f} / \mathrm{f}} \mathrm{Cdh} 5 \mathrm{Cre}$ mice at a late stage (20 weeks) (D) of intracranial aneurysm formation. In (C), sections were also stained for the endothelial cell marker CD31. Nuclear staining with DAPI (blue) is also shown in merged images. Representative images are shown ( $n=4$ mice for each genotype and time point from three independent experiments). Scale bars, $30 \mu \mathrm{m}$ (C) and $40 \mu \mathrm{m}$ (D). (E and F) Intracranial aneurysm incidence (E) and macrophage infiltration (F) in Ptger $2^{\mathrm{f} / \mathrm{f}}$ Lyz2 2 re and Ptger $2 / \mathrm{f} / \mathrm{C}$ Ch5Cre mice 5 months after intracranial aneurysm induction. The numbers of animals used are shown in parentheses. Statistical analysis was performed using Fisher's exact test (E) or a Mann-Whitney test (F). Bars in (F) indicate means \pm SEM.

\section{EP2 signaling directly activates NF-KB and synergistically induces NF-kB-dependent gene expression with TNF- $\alpha$ through a PGE $_{2}$-EP2-NF-kB-COX-2-positive feedback loop} directly induce NF- $\kappa \mathrm{B}$ activation and to determine the underlying mechanism, we generated HEK293 cells stably expressing mouse EP2 (fig. S12A) and confirmed EP2 expression by immunohistochemistry (fig. S12B) and by increases in
To examine whether EP2 stimulation could 
A
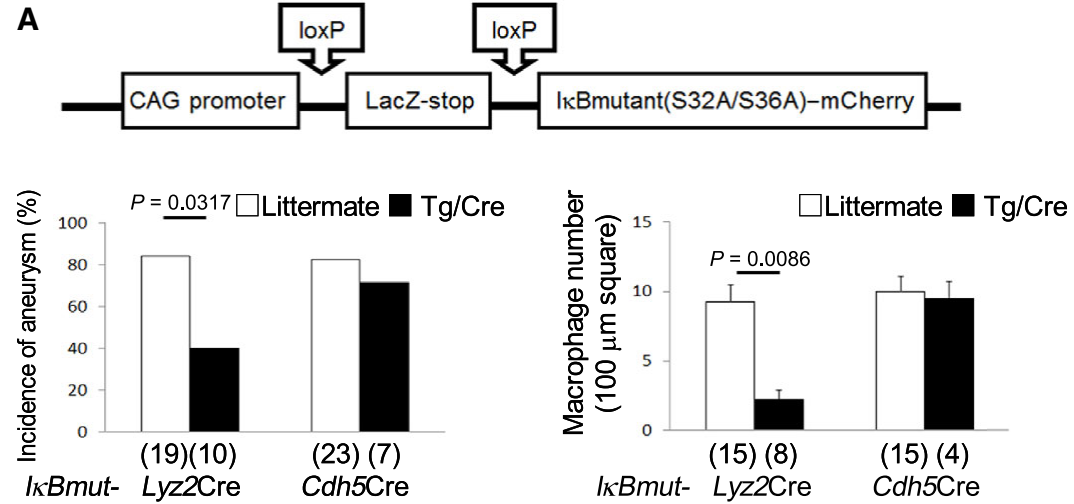

B

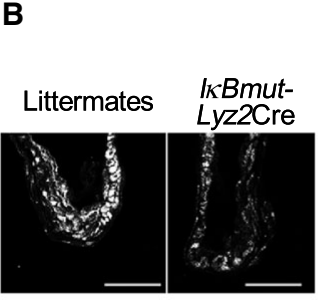

$p-p 65$

D
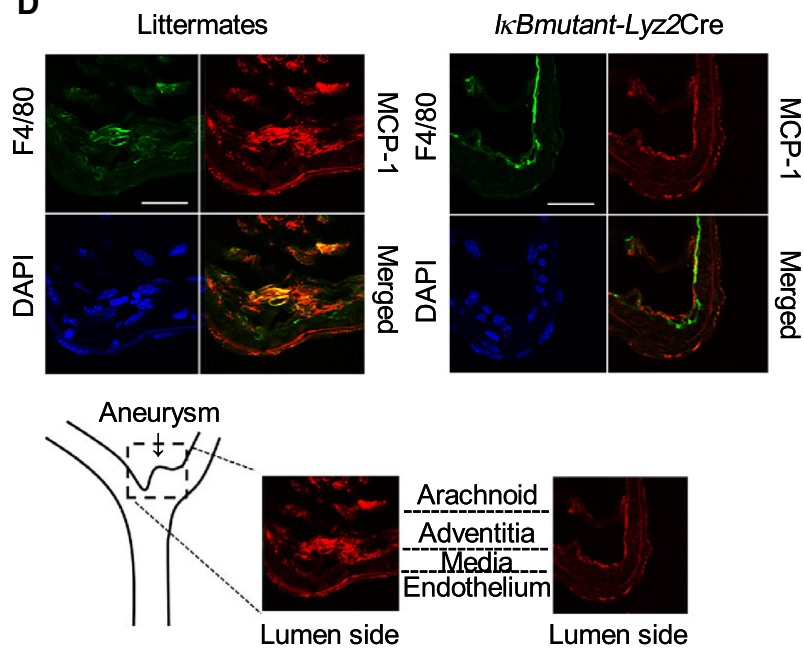

Fig. 3. NF-кB activation in macrophages is required for sustained inflammation in intracranial arteries and intracranial aneurysm formation. (A) Intracranial aneurysm incidence and macrophage infiltration in macrophage-specific Lyz2-Cre and endothelial cell-specific Cdh5-Cre IкB mutant mice 5 months after intracranial aneurysm induction. The $І \kappa B$ mutant construct, the intracranial aneurysm incidence, and macrophage infiltration are shown. The numbers of animals used are shown in parentheses. Statistical analysis was performed using Fisher's exact test (left) or a Mann-Whitney test (right). Bars in the right panel indicate means \pm SEM. (B and C) Suppression of NF- $\mathrm{KB}$ activation (B) and increases in $\mathrm{C} C \mathrm{C} 2$ and Ptgs2 expression $(C)$ in intracranial aneurysm lesions in $I \kappa B$ mutant-Lyz2Cre mice. Samples were collected for analyses from mice that were not operated on as a control $(0 \mathrm{M})$ and mice 5 months after intracranial aneurysm induction (5 M). Immunostaining for the phosphorylated form of p65 (Ser ${ }^{536}$; p-p65) representative of five samples from each genotype is shown in (B), and quantitative RT-PCR analyses for CCl2 and Ptgs2 are shown in (C) ( $n=8$ littermates or $4 \mathrm{Tg} / \mathrm{Cre}$ mice; Kruskal-Wallis test). Scale bars, $30 \mu \mathrm{m}$. (D) MCP-1 abundance in intracranial aneurysm walls in $/ \kappa B$ mutant-Lyz2Cre mice. Immunostaining for F4/80 (green) and MCP-1 (red), nuclear staining by DAPI, and merged images are shown ( $n=5$ mice for each genotype from three independent experiments). Scale bars, $20 \mu \mathrm{m}$. In the lower panels, sketch of the aneurysm lesion is shown as reference. adenosine $3^{\prime}, 5^{\prime}$-monophosphate (cAMP) in response to the selective EP2 agonist ONO-AE1-259 (fig. S12C) (28). Stimulation with ONO-AE1-259 significantly increased the nuclear translocation of the canonical NF- $\kappa \mathrm{B}$ subunits $\mathrm{p} 50$ and $\mathrm{p} 65$, but not that of c-Rel (Fig. 4A). ONO-AE1-259 also induced the NF- $\kappa \mathrm{B}-$ dependent expression of an NF- $\kappa \mathrm{B}$ reporter in HEK293-EP2 cells (fig. S13A). These findings are consistent with our previous in vivo findings showing that the p50-p65 complex is activated in intracranial arteries after aneurysm induction (15) and that Ptger2 deficiency decreases p65 phosphorylation in intracranial arteries (18). We next examined whether EP2 signaling affected phosphorylation of $\mathrm{Ser}^{337}$ in p50, an event that is critical for DNA binding $(29,30)$. ONO-AE1-259 increased the phosphorylation of this site in a time- and concentration-dependent manner (Fig. 4B), and this was mimicked by the cAMP analog dibutyryl-cAMP (db-cAMP), and inhibited by the protein kinase A (PKA) inhibitor H-89 (fig. S13B), indicating that EP2 simulation mobilizes the cAMPPKA pathway to phosphorylate the $\mathrm{p} 50$ subunit. ONOAE1-259 also increased phosphorylation of NF- $\kappa$ B p65 at Ser $^{536}$ in a time-dependent manner (Fig. 4C).

These results suggest that EP2 signaling activates the canonical NF- $\kappa \mathrm{B}$ signaling cascade by inducing the phosphorylation of the p50 and p65 subunits. However, this activation was much weaker than that induced by cytokines such as TNF- $\alpha$ (Fig. 4C and fig. S13, A and C), which led us to hypothesize that $\mathrm{NF}-\kappa \mathrm{B}$ activation by EP2 stimulation synergizes with other signaling pathways to amplify expression of $\mathrm{NF}-\kappa \mathrm{B}$ target genes. We chose to test this synergism hypothesis using TNF- $\alpha$ because TNF- $\alpha$ signaling also contributes to intracranial aneurysm formation (31-33). Treatment of primary macrophages isolated from the intraperitoneal cavity with ONO-AE1-259 and TNF- $\alpha$ potently amplified the expression of TNF$\alpha$-induced, NF- $\kappa \mathrm{B}$-dependent genes, including Il6 [which encodes interleukin-6 (IL-6)], Il1b (which encodes IL-1 $\beta$ ), Ptgs2, and Nos2 (which encodes inducible nitric oxide synthase) (Fig. 4D and fig. S13D). Consistent with NF-אB activation by EP2 stimulation, NF- $\kappa$ B inhibitor IV (34-36) significantly attenuated the amplifying effect of ONO-AE1-259 on TNF- $\alpha$-induced gene expression (fig. S14, A to D). We further examined the molecular mechanism underlying the synergy between EP2 and TNF- $\alpha$ by using HEK293-EP2 cells expressing a Ptgs 2 promotercontaining luciferase reporter. We found that ONOAE1-259 increased luciferase activity and Ptgs2 expression synergistically with TNF- $\alpha$ (Fig. $4 \mathrm{E}$ and fig. S15A). Because both EP2 and TNF- $\alpha$ signaling activate NF- $\kappa \mathrm{B}$, and the Ptgs 2 promoter contains two consensus NF- $\kappa \mathrm{B}$ binding sites (the distal NF- $\kappa \mathrm{B}$ site is located about -447 position and the proximal NF- $\kappa \mathrm{B}$ site is about -223 position from the transcription initiation site), we examined NF- $\mathrm{NB}$ binding sites that responded to each signaling stimulus. ONO-AE1-259 
A

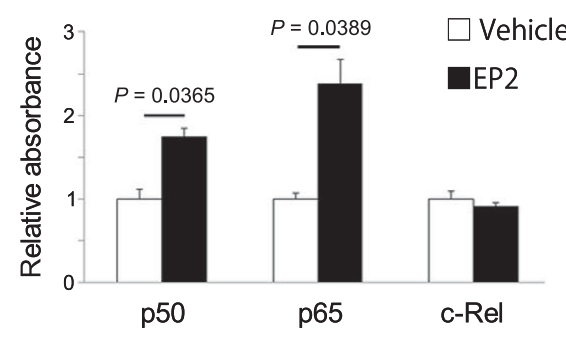

C

EP2 (min) $\quad 0 \quad 20 \quad 60 \quad 120 \quad 240 \quad$ TNF

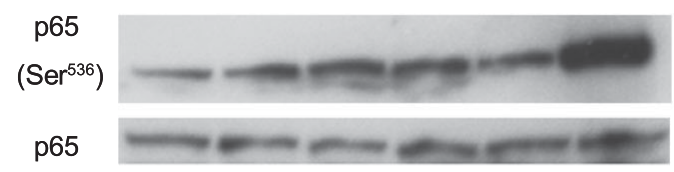

$\alpha$-Tubulin

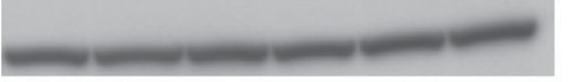

E

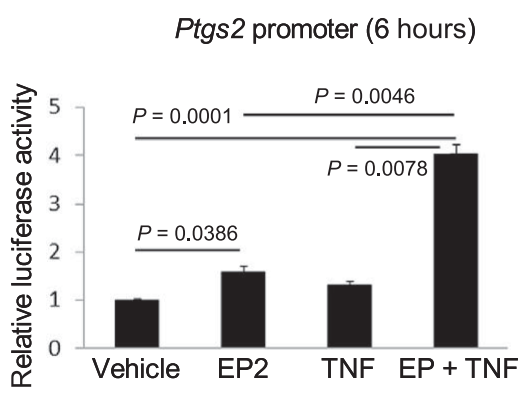

G

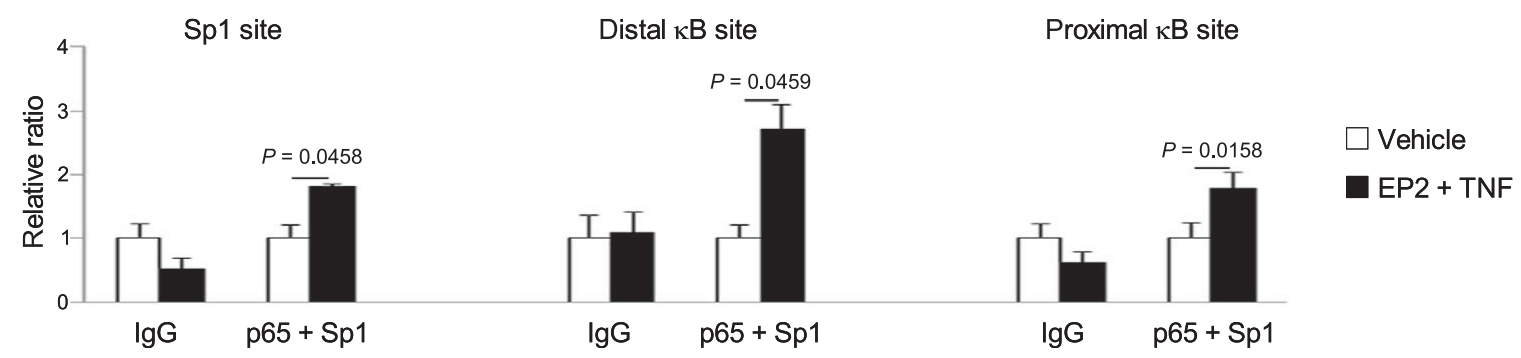

D

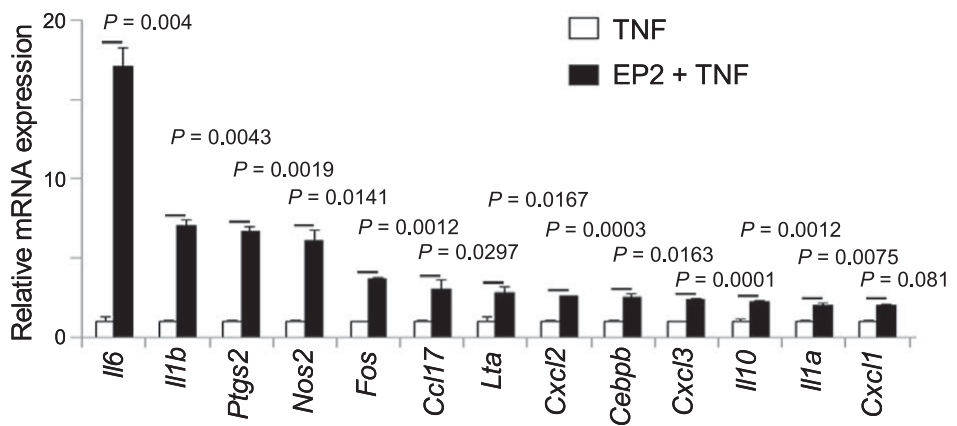

$\mathbf{F}$

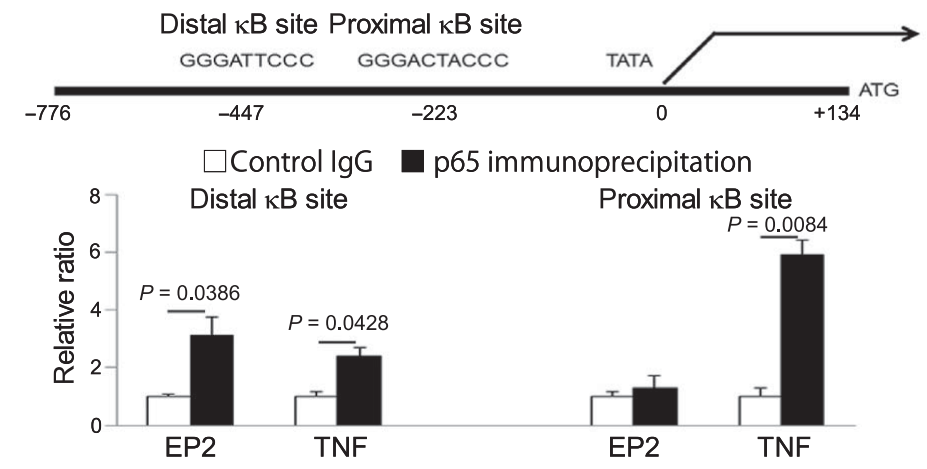

Fig. 4. Synergistic activation of NF-KB and NF-KB-induced gene expression by EP2 and TNF- $\alpha$ signaling. (A to C) Nuclear translocation and time- and dosedependent phosphorylation of p50/p65 NF-kB subunits after EP2 stimulation. HEK293 cells stably expressing EP2 (HEK293-EP2 cells) were stimulated with ONO-AE1-259 for the indicated times or at the indicated concentrations. (A) Nuclear translocation was assessed using a DNA binding enzyme-linked immunosorbent assay ( $n=3$ biological replicates, Mann-Whitney test). (B) p50 (Ser ${ }^{337}$ ) phosphorylation was analyzed, and a representative Western blot is shown with $\alpha$-tubulin as an internal control ( $n=3$ independent experiments). (C) p65 (Ser ${ }^{536}$ ) phosphorylation was analyzed with $\alpha$-tubulin as an internal control and with TNF- $\alpha$ (TNF) stimulation as a positive control ( $n=3$ independent experiments). (D) Peritoneal macrophages were stimulated with TNF- $\alpha$ alone or with ONO-AE1-259, and the expression of NF-kB-regulated genes was analyzed by PCR array analysis ( $n=3$ biological replicates, Mann-Whitney test). (E to G) HEK293-EP2 cells transfected with the Ptgs2 promoter-luciferase construct were stimulated with TNF- $\alpha$, ONO-AE1-259, or both. Cells were assayed for luciferase activity ( $n=5$ biological replicates, Kruskal-Wallis test) (E), ChIP assay using an antibody specific for the p65 subunit ( $n=3$ biological replicates, Mann-Whitney test) (F), and Re-ChIP assay using antibodies specific for p65 and Sp1 ( $n=6$ biological replicates, Mann-Whitney test) (G). 
enhanced the luciferase activity of a construct containing the distal NF$\kappa \mathrm{B}$ site, and mutation or deletion of this site significantly attenuated the ONO-AE1-259-induced increase in Ptgs2 promoter activity (fig. S15B). In contrast, TNF- $\alpha$ stimulation enhanced the luciferase activity of the construct containing the proximal NF- $\mathrm{BB}$ site, and deletion of this site attenuated TNF- $\alpha$ action (fig. S15C). Consistent with these findings, chromatin immunoprecipitation (ChIP) analysis revealed that ONOAE1-259 treatment resulted in selective binding of p65 to the distal NF$\kappa \mathrm{B}$ site, whereas p65 activated by TNF- $\alpha$ signaling bound to the proximal $\mathrm{NF}-\kappa \mathrm{B}$ site to a greater extent (Fig. $4 \mathrm{~F}$ ). Although the precise mechanism by which NF- $\kappa \mathrm{B}$ activated by different signaling events leads to binding to different NF- $\kappa \mathrm{B}$ sites is not clear, we suspected that differences in the composition of the transcription factor complex could be important. We noted that both the DNA binding and transcriptional activity of Sp1 were activated by TNF- $\alpha$ stimulation but not by EP2 stimulation (fig. S16, A and B). Pharmacological inhibition of Sp1 using mithramycin or depletion of $S p 1$ by RNA interference (RNAi) inhibited the enhancing effect of TNF- $\alpha$ or the synergistic effect of EP2 and TNF- $\alpha$ on Ptgs 2 promoter-driven luciferase activity; in contrast, overexpression of Sp1 enhanced luciferase activity under these conditions (fig. S16, C to E). These data suggest the formation of a transcription factor complex containing p50, p65, and Sp1. To corroborate this idea, we performed Re-ChIP assays in which transcriptional complexes were first immunoprecipitated with an antibody specific for p65 and then with an antibody for Sp1. Immunoprecipitation of the Ptgs 2 promoter DNA was then examined by quantitative PCR analysis. Chromatin fragments corresponding to the Sp1 binding site, the distal NF- $\kappa \mathrm{B}$ site, and the proximal NF- $\kappa \mathrm{B}$ site of the Ptgs 2 promoter were all significantly enriched by sequential immunoprecipitation (Fig. 4G). In addition, the DNA binding activity of Spl was increased in rat intracranial arterial samples 1 month after intracranial aneurysm induction without changes in its mRNA expression (fig. S16, F and G). Together, these data suggested the formation of a transcription factor complex containing p50-p65-Sp1 on the promoter region of Ptgs 2 in vitro and in vivo. Given that $\mathrm{COX}-2$ initiates $\mathrm{PGE}_{2}$ biosynthesis, these findings revealed a positive feedback loop of the $\mathrm{PGE}_{2}-\mathrm{EP} 2-\mathrm{NF}-\mathrm{\kappa B}-$ COX-2 pathway to amplify PG-mediated, NF- $\kappa \mathrm{B}-$ dependent inflammation by induction of COX-2. The presence of such an amplification loop has been suggested for intracranial aneurysm formation in vivo $(9,13,18)$ and is consistent with our findings (Figs. 2 and 3).

\section{EP2 signaling stabilizes $\mathrm{C} / 2$ mRNA by activating the RNA binding protein HuR}

Because MCP-1 induction in intracranial aneurysm lesions could lead to the formation of a self-amplification loop that exacerbates inflammation by recruiting more macrophages into the lesion, thereby facilitating intracranial aneurysm progression (Fig. 3) $(7,9,19,27)$, we next examined whether EP2 signaling could induce $\mathrm{Ccl} 2$ (which encodes MCP-1) expression synergistically with TNF- $\alpha$, as was observed for Ptgs2 expression. ONO-AE1-259 stimulation alone failed to induce Ccl 2 mRNA expression, but it significantly enhanced the expression of the gene when added with TNF- $\alpha$ compared to cells that were stimulated only with TNF- $\alpha$ (Fig. 5A). Ccl2 mRNA is stabilized by the RNA binding protein $\mathrm{HuR}$ (37). To examine whether posttranscriptional modifications might be involved in the amplifying action of ONOAE1-259 on $\mathrm{Ccl} 2$ expression, $\mathrm{Ccl} 2 \mathrm{mRNA}$ expression was induced by TNF- $\alpha$ in HEK293-EP2 cells before the transcriptional inhibitor actinomycin D was added. We found that ONO-AE1-259 significantly delayed the decay of $\mathrm{Ccl} 2 \mathrm{mRNA}$ (Fig. 5B) but not that of Ptgs 2
mRNA (fig. S17A), suggesting that EP2 signaling stabilized Ccl2 mRNA to amplify inflammation through macrophage infiltration. To further confirm the stabilizing effect of EP2 signaling on Ccl2 mRNA expression, we performed luciferase reporter assays using a construct containing the $3^{\prime}$ untranslated region (3'UTR) of the human $\mathrm{Ccl} 2 \mathrm{mRNA}$ [355 base pairs (bp)] (Fig. 5C); costimulation with interferon- $\gamma$ (IFN- $\gamma$ ) and TNF- $\alpha$ were used as positive controls (37). Consistent with the above results, ONO-AE1-259 significantly enhanced the activity of the luciferase reporter in EP2-expressing HEK293 cells (Fig. 5C). We next assessed the involvement of HuR in the effect of ONO-AE1-259. Depletion of Elavl1 (which encodes HuR) by RNAi or removal of the HuR-binding site from the $3^{\prime} \mathrm{UTR}$ of $\mathrm{Ccl} 2$ in the luciferase construct attenuated the effect of ONO-AE1-259 on the promoter activity of the Ccl 2 luciferase reporter (Fig. 5, D and E, and fig. S17B), confirming the involvement of $\mathrm{HuR}$ in the effect of EP2 signaling on the stabilization of Ccl2 mRNA. HuR translocates from the nucleus to the cytoplasm to stabilize its target mRNAs (38-40). As expected from the above results, nuclear-to-cytoplasmic translocation of $\mathrm{HuR}$ was induced by ONO-AE1-259 treatment, as shown by Western blot analysis of subcellular fractions (fig. S17C). We then examined whether EP2 stimulation promoted the binding of HuR to the $3^{\prime} \mathrm{UTR}$ of the Ccl2 mRNA by performing quantitative RT-PCR analysis on HuR immunoprecipitates of nuclear extracts prepared from HEK293-EP2 cells. We found that Ccl 2 mRNA was enriched in HuR immunoprecipitates compared with control immunoglobulin $\mathrm{G}$ ( $\mathrm{IgG})$ immunoprecipitates and that ONOAE1-259 treatment increased the enrichment of $\mathrm{Ccl} 2$ more than 10-fold (Fig. 5F), confirming that EP2 signaling facilitated HuR binding to the 3'UTR of the $C c l 2$ gene. p38 mitogen-activated protein kinase (MAPK) signaling is involved in the nuclear-to-cytoplasmic translocation of $\mathrm{HuR}$ (38). EP2 signaling activated p38 MAPK (fig. S18, A and B), and the p38 MAPK inhibitors SB202190 or SB239063 attenuated the effect of ONO-AE1-259 on the activity of the Ccl2 luciferase reporter (fig. S17D). This effect was mimicked by leptomycin B, which inhibits nuclear export of proteins (fig. S17D). Together, these data support a role for p38 MAPK activated by EP2 signaling in the HuR-mediated stabilization of $\mathrm{Ccl} 2 \mathrm{mRNA}$.

\section{Oral administration of the selective EP2 antagonist PF- 04418948 suppressed intracranial aneurysm progression}

Because EP2 functions as a node for inflammatory responses responsible for intracranial aneurysm formation, we next examined the potential of pharmacological manipulation of EP2 as a therapeutic measure to treat intracranial aneurysms. We noted that, in the microarray analysis using the ring of Willis from rats with induced intracranial aneurysms, Ptger 2 was among the genes with the greatest increase in expression in lesions compared to normal (table S1 and data file S1), which further supports the potential of EP2 as a therapeutic target. The selective EP2 antagonist PF-04418948 (41) dose-dependently decreased the size of induced intracranial aneurysms at 3 weeks (Fig. 6A), and furthermore, it reduced degenerative changes in the media, such as thinning of the medial smooth muscle cell layer and preservation of $\alpha$-smooth muscle actin-positive cells, without significantly affecting systemic blood pressure (fig. S19, A and B). Pharmacological inhibition of EP2 also prevented increases in the phosphorylation of NF- $\kappa \mathrm{B}$ p65 at $\mathrm{Ser}^{536}$ and in the abundance of COX-2 and MCP-1 (Fig. 6B) and suppressed macrophage infiltration into intracranial aneurysm lesions (Fig. 6C).

Because intracranial aneurysms in most patients are usually found during magnetic resonance imaging (MRI) examination of the brain $(6,42)$, a drug therapy for intracranial aneurysms must be able to target preexisting intracranial aneurysms. In patients with unruptured 


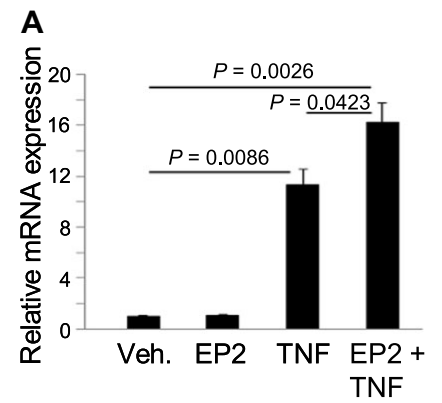

C
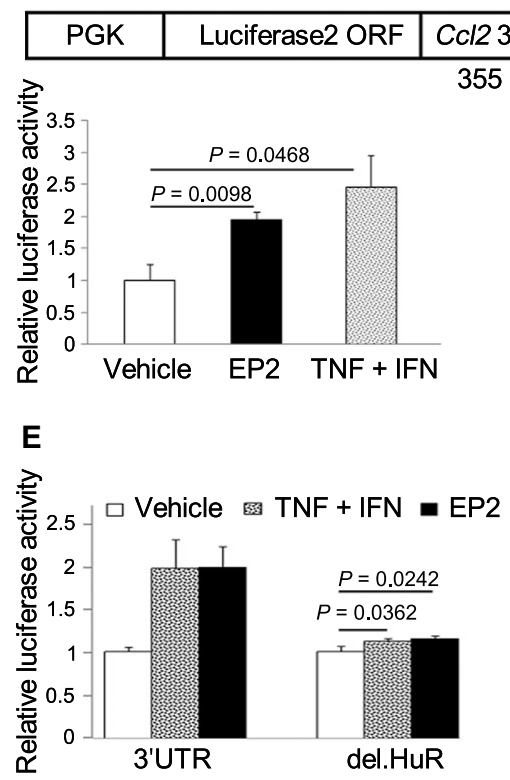

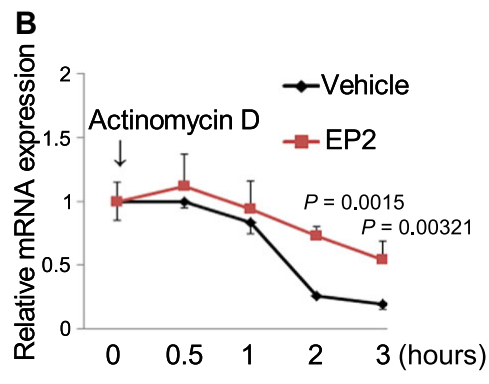

D
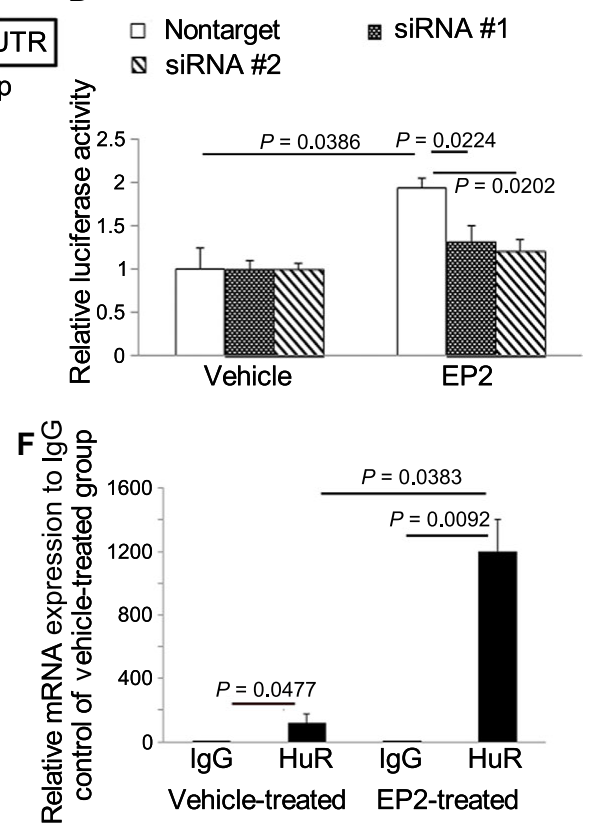

Fig. 5. EP2 mediates stabilization of Ccl2 mRNA through HuR activation. (A) HEK293-EP2 cells were stimulated with ONO-AE1-259, TNF- $\alpha$, or both, and CCl2 mRNA expression was examined by quantitative $\mathrm{RT}-\mathrm{PCR}$ (means $\pm \mathrm{SEM}, n=3$ biological replicates, Kruskal-Wallis test). (B) Actinomycin D was added to HEK293-EP2 cells stimulated with TNF- $\alpha$ and either vehicle or ONO-AE1-259. The decrease in Ccl2 mRNA was examined using quantitative RT-PCR ( $n=5$ biological replicates, Mann-Whitney test). (C to E) HEK293-EP2 cells transfected with the luciferase construct harboring the $\mathrm{CCl} 23^{\prime} \mathrm{UTR}$ were treated with ONO-AE1-259 using costimulation of IFN- $\gamma$ (IFN) and TNF- $\alpha$, and luciferase activity was measured $[(C), n=4$ biological replicates]. The contribution of HuR binding to EP2-mediated stabilization of luciferase mRNA was examined using Elavl1 (which encodes HuR)-targeted small interfering RNAs (siRNAs) [(D), nontarget siRNA and two Elavl1-targeted siRNAs (siRNA \#1 and siRNA \#2), $n=4$ biological replicates] and deletion of the HuR-binding site [(E), del.HuR, $n=5$ biological replicates, Kruskal-Wallis test]. (F) Binding of HuR to the Ccl2 3'UTR examined by RNA immunoprecipitation with an antibody specific for HuR or lgG as a negative control in HEK293-EP2 cells treated with either vehicle or ONO-AE1-259 ( $n=4$ biological replicates, Kruskal-Wallis test). potential of EP2 as a therapeutic target for intracranial aneurysms and suggest that an EP2 antagonist could be a promising drug candidate for treatment of human intracranial aneurysms.

\section{DISCUSSION}

Here, we have used clinical tissue samples taken from intracranial aneurysm patients, transgenic animal models, and transfected cultured cells to examine the mechanism underlying intracranial aneurysm formation. We focused on the COX-2-PGE $2-\mathrm{EP} 2$ signaling pathway, because we have previously found that Ptger 2 deficiency suppresses intracranial aneurysm formation and that NF- $\mathrm{KB}-$ mediated inflammation occurs in the mouse intracranial aneurysm model (18). We found a positive correlation between EP2 abundance and macrophage infiltration in the human intracranial aneurysm tissue samples (Fig. 1). We discovered that EP2 signaling in macrophages sustained the NF- $\kappa \mathrm{B}$-mediated inflammation in the lesion and induced intracranial aneurysm formation. NF- $\kappa \mathrm{B}$ activation in macrophages was crucial for intracranial aneurysm formation and for the inflammatory response. Our study also revealed that EP2 signaling directly activated NF- $\kappa \mathrm{B}$ and that this signaling pathway synergized with TNF- $\alpha$ to induce expression of various proinflammatory genes (Fig. 7). Notably, EP2 and TNF- $\alpha$ synergized in the expression of Ptgs 2 at the transcriptional level, and EP2 activation further enhanced TNF- $\alpha$-induced expression of $\mathrm{Ccl} 2$ posttranscriptionally through HuR-mediated RNA stabilization (Fig. 7). These findings suggest that the COX-2-PGE 2 -EP2 signaling forms a positive feedback loop through the activation of NF- $\kappa \mathrm{B}$ to amplify and sustain inflammation. Enhanced MCP-1 abundance in macrophages also amplifies inflammation by recruiting more macrophages to the lesion. The COX-2-PGE $2-\mathrm{EP} 2$ signaling in macrophages thus functions as an inflammatory node in intracranial aneurysm formation. Because the abundance of both COX-2 and mPGES-1, an important enzyme synthesizing $\mathrm{PGE}_{2}$ downstream of $\mathrm{COX}-2$, is increased in ruptured intracranial aneurysms from human patients (43), this signaling cascade could intracranial aneurysms, the annual rate of rupture is positively correlated with the size of the aneurysm (6). We therefore examined the therapeutic effects of PF-04418948 on preexisting intracranial aneurysms with prevention of further enlargement as an endpoint. Rate of aneurysm rupture was not chosen as the endpoint because only a very small number of the intracranial aneurysms induced in this rat model spontaneously rupture (about $1 \%$ per month) (8). In the vehicle-treated group, intracranial aneurysms progressively enlarged during the followup period (Fig. 6D). In contrast, PF-04418948 significantly suppressed the further enlargement of the induced intracranial aneurysms without influencing systemic blood pressure (Fig. 6D and fig. S19C). Histologically, treatment with PF-04418948 suppressed media thinning (fig. S19D) and macrophage infiltration (Fig. 6E). These data corroborate the be a promising target for drugs to treat intracranial aneurysms to prevent not only their formation but also their rupture. Nonsteroidal antiinflammatory drugs (NSAIDs), such as COX inhibitors, may therefore be good candidate drugs for intracranial aneurysm treatment. However, some case-control studies have reported that NSAIDs prevent the rupture of intracranial aneurysms (which causes subarachnoid hemorrhages) $(44,45)$, but others have reported that they facilitate the rupture of these aneurysms (46). These discrepancies may be due to the diverse actions of these types of drugs; they exert not only antiinflammatory actions but also anti-platelet actions, and some, particularly selective COX-2 inhibitors, even increase cardiovascular risk (33). NSAIDs have also considerable adverse effects, such as gastrointestinal toxicity. These adverse effects would make it difficult to use NSAIDs 
A
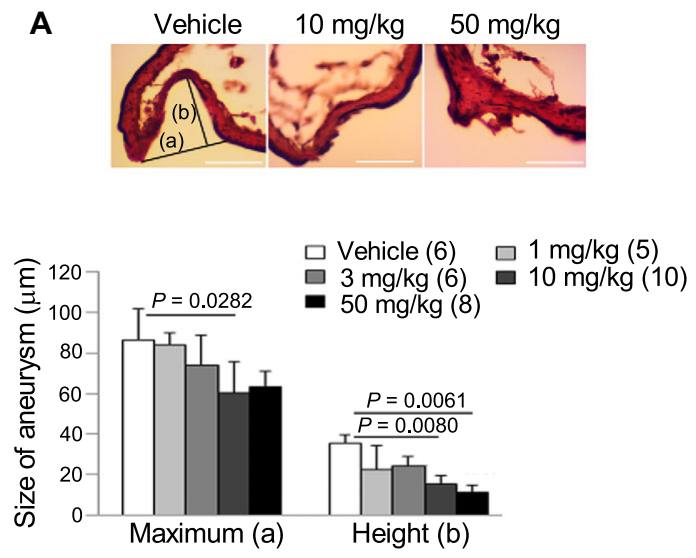

B

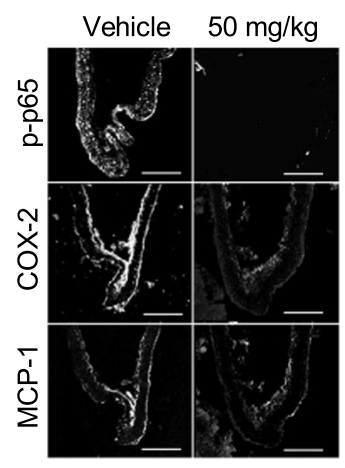

C

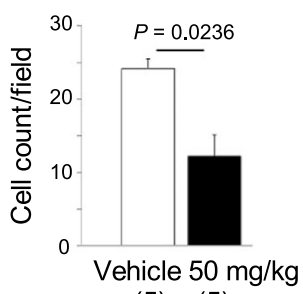

(5) (5)

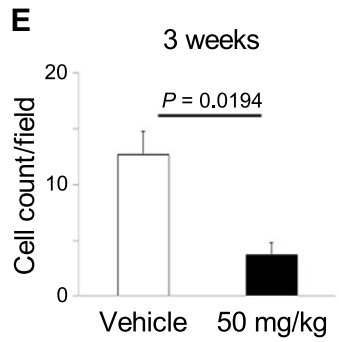

(4)
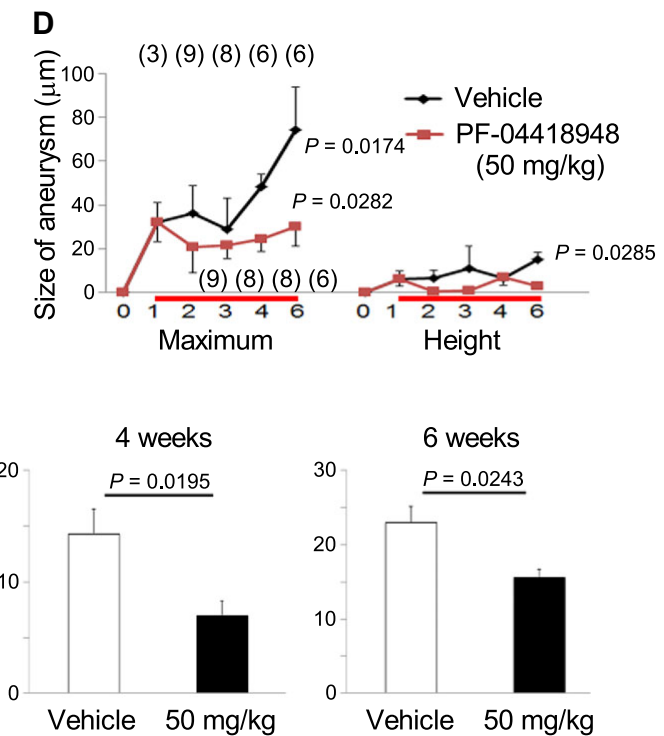

(6)

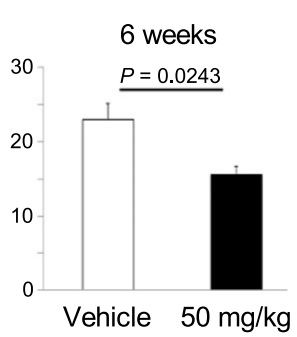

(4)
(6)

Fig. 6. Suppression of intracranial aneurysm development and enlargement by administration of the EP2 antagonist PF-04418948 in rats through suppression of inflammatory responses. (A) Representative images of Elastica van Gieson staining and quantitative measurement of intracranial aneurysms in rats treated with the indicated doses of PF-04418948. Scale bars, $50 \mu \mathrm{m}$. a, maximum width in the aneurysm wall; $b$, height of the aneurysm. The number of animals examined is shown in parentheses. Statistical analysis was performed using a Kruskal-Wallis test. (B and C) Immunostaining for p-p65, COX-2, and MCP-1 in rats with intracranial aneurysms treated with vehicle or PF-04418948 [(B), $n=3$ rats for each treatment] and the number of infiltrated macrophages found in intracranial aneurysm lesions $[(\mathrm{C}), n=5$ rats for each treatment, Mann-Whitney test]. Scale bars, $50 \mu \mathrm{m}$. (D and E) One week after intracranial aneurysm induction, rats were treated by oral administration of either vehicle (black line) or PF-04418948 (red line) for the indicated periods. Intracranial aneurysm size was examined (D), and the number of infiltrated macrophages in the lesion was counted (E). The number of animals used is shown in parentheses. Statistical analysis was performed using a Mann-Whitney test.

as prophylactic drugs to control the macrophage-derived inflammatory signals in intracranial aneurysms. Given the high incidence of intracranial aneurysms in the general public and the severity of resultant subarachnoid hemorrhage after rupture, more selective and safer alternatives to NSAIDs or COX-2 inhibitors are therefore desired. The present study demonstrated that administration of the selective EP2 antagonist PF04418948 significantly suppressed not only the development of intracranial aneurysms but also the enlargement of preexisting intracranial aneurysms in rats. In light of a large observational study conducted in Japan that demonstrated that the annual rupture risk of unruptured in- tracranial aneurysms positively correlates with size (6), an EP2 antagonist is therefore a promising candidate to treat human intracranial aneurysms. Furthermore, the rate of subarachnoid hemorrhage after elastase-induced intracranial aneurysm (47) is increased in mPGES-1-deficient mice, compared to wild-type mice, although the incidence of intracranial aneurysms is similar between the two genotypes. Aspirin, but not $\mathrm{PGE}_{2}$, could ameliorate the increase in subarachnoid hemorrhage (47), suggesting the involvement of a PG or PGs other than $\mathrm{PGE}_{2}$ in the rupture of intracranial aneurysm, which supports the potential superiority of EP2 over mPGES-1 as a therapeutic target to prevent rupture of intracranial aneurysms. However, development of a selective EP2 antagonist to prevent rupture of preexisting intracranial aneurysms will require the establishment of an animal model in which intracranial aneurysms rupture more frequently.

Numerous previous studies using either human tissue samples or animal models of intracranial aneurysms have shown that macrophages are major inflammatory cells that infiltrate the intracranial aneurysm lesions $(19,21,22,27,48,49)$. A role for MCP-1 in macrophage recruitment in intracranial aneurysm formation has been suggested by studies using mice deficient in MCP-1 or rats in which MCP-1 is inhibited with a dominant negative form $(19,27)$. Here, we identified intracranial aneurysms as a macrophage-mediated inflammatory disease of intracranial arteries by demonstrating the crucial role of the COX-2-PGE 2 -EP2-NF-кB-MCP-1 pathway in macrophages in intracranial aneurysm formation. This concept is pivotal not only for basic research aimed at understanding the pathogenesis of intracranial aneurysms but also for translational research in applying these findings to the clinical setting, and our results suggest the therapeutic value of targeting this cell type in intracranial aneurysms. The selective contribution of the COX-2 signaling pathway in macrophages to the pathogenesis of vascular disease has also been demonstrated in atherogenesis, in which macrophage-specific deletion of COX-2 ameliorates atherogenesis in hyperlipidemic mice (50), suggesting that drugs targeting macrophages can be used in other vascular diseases. Macrophage activation may also be used as a surrogate marker of inflammation in intracranial aneurysm walls to distinguish between rupture-prone intracranial aneurysms and others with a lower probability of rupture. Our findings support those obtained from noninvasive MRI of macrophages in the intracranial aneurysm walls in patients administered ultrasmall superparamagnetic iron oxide (USPIO) (51). This approach thus allows for qualitative diagnosis of intracranial aneurysms before surgical intervention and may thus be a breakthrough in the treatment of intracranial aneurysms, because, currently, the annual rupture risk of incidentally found intracranial aneurysms is estimated only from morphological aspects, such as size or shape. Other confounding factors, such as family history or racial background, also increase the risk of rupture (5). As a corollary to the current clinical situation, small intracranial 


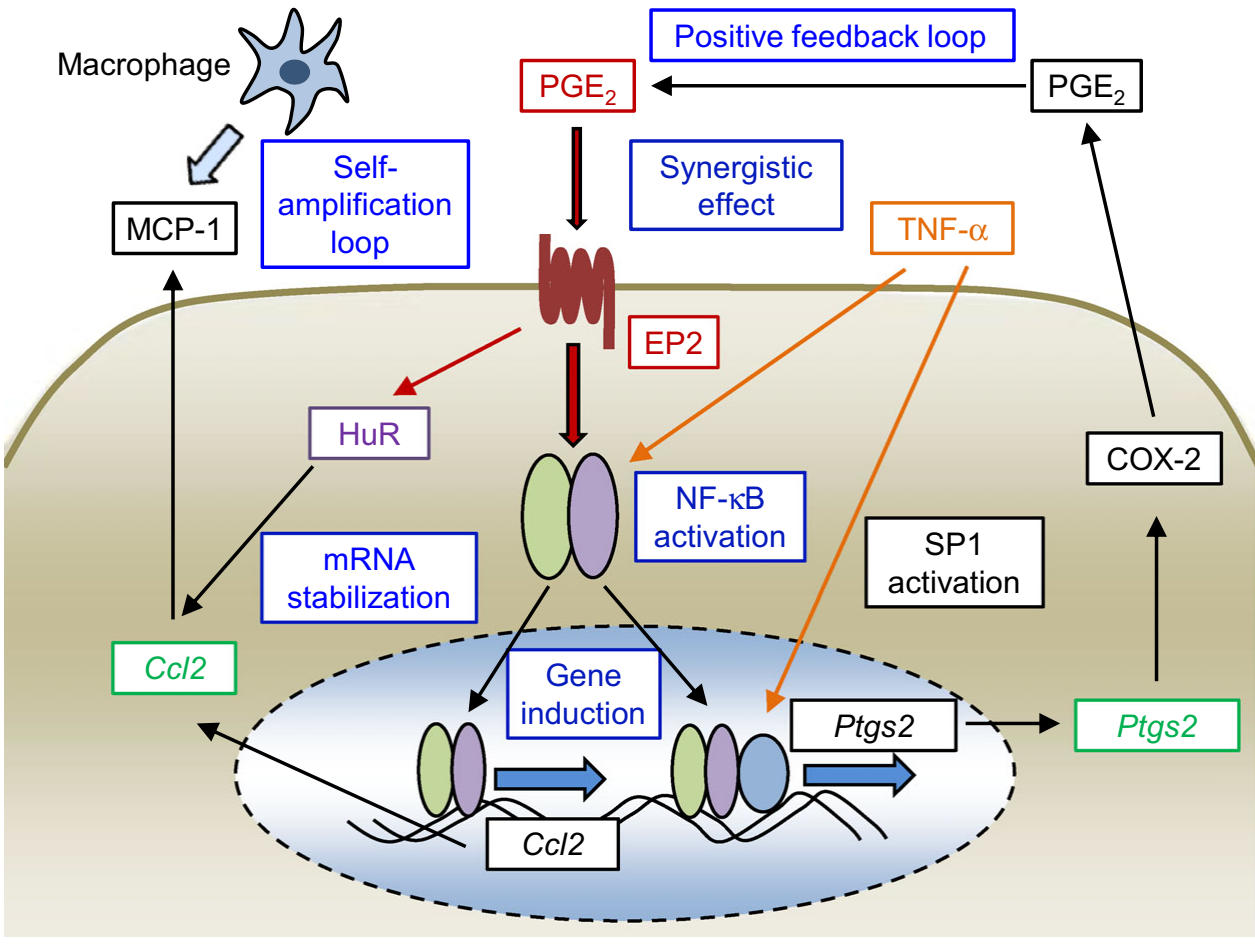

Fig. 7. Proposed roles and mechanisms of $\mathrm{PGE}_{2}-\mathrm{EP2}$ signaling to regulate chronic inflammation. $E P 2$ signaling directly activates NF-KB and synergizes with TNF- $\alpha$ to induce expression of various proinflammatory genes. For Ptgs2, this synergism occurs at the transcriptional level. For CCl2, EP2 activation further enhances TNF- $\alpha$-induced expression of this gene posttranscriptionally through HuR-mediated RNA stabilization.

further amplification of macrophage accumulation and macrophage-mediated inflammation. The $\mathrm{PGE}_{2}$-EP2 signaling pathway in macrophages thus critically contributes in amplifying and sustaining inflammation in intracranial aneurysm lesions. In this sense, we can regard an intracranial aneurysm as a chronic inflammatory disease regulated by $\mathrm{PGE}_{2}-\mathrm{EP} 2$ signaling. Presumably, this may be a common pathogenesis in other chronic diseases; we have found a similar amplifying loop involving $\mathrm{PGE}_{2}$-EP2 signaling in colitis-associated cancer (54). Therefore, the present findings may contribute to our understanding of the pathogenesis of various chronic inflammatory diseases. EP4, but not EP2, signaling facilitates disease progression, including rupture of aortic aneurysm (55-57), and thus, our results imply that the PG system regulates disease pathogenesis through different receptor subtypes, presumably according to their microenvironment.

A remaining question is how macrophages are recruited to the adventitia and activated to initiate the formation of the intracranial aneurysm lesions. Analysis from the computational fluid dynamics field suggests that high wall shear stress acts on endothelial cells to trigger intracranial

aneurysms sometimes rupture before surgical treatment, and large intracranial aneurysms with a low probability of rupture are surgically treated with the risk of attendant complications. Further studies in humans will be required to carefully examine whether MRI of macrophage infiltration with USPIO reflects aneurysm enlargement or risk of rupture, and if insufficient, imaging particles that provide higher resolution and contrast should be developed. Using such a technique, we can envisage being able to sequentially assess the inflammatory status in intracranial aneurysm lesions in a less invasive manner and allow for the effect of investigational drugs to be studied over a shorter period of time, which could greatly facilitate the development of novel drugs to treat intracranial aneurysms. In addition, macrophage-specific targeting of therapeutic drugs by applying this nanoparticle technique as a drug delivery vehicle may contribute to the development of a safer and more effective drug therapy.

Chronic inflammation underlies various chronic intractable diseases, such as cancer (52), vascular diseases including atherosclerosis (53), autoimmune diseases, and neurodegenerative diseases. We suggest that the PG system can contribute to the establishment of chronic inflammation. In this model, PGs function synergistically with cytokines and form a positive feedback loop to amplify and sustain inflammation (9). Here, we found a positive feedback loop involving COX-2-PGE 2 -EP2$\mathrm{NF}-\kappa \mathrm{B}$ signaling in macrophages present in intracranial aneurysms. Furthermore, the $\mathrm{PGE}_{2}-\mathrm{EP} 2$ signaling pathway functions as a "cytokine amplifier" to amplify the TNF- $\alpha$-induced expression of various inflammationassociated genes, thereby exacerbating TNF- $\alpha$-evoked inflammation. In the case of Ptgs2 expression, the two pathways function together to facilitate the formation of the transcription factor complex including NF- $\mathrm{\kappa B}$ and $\mathrm{Sp} 1$, and in the case of $\mathrm{Ccl} 2, \mathrm{PGE}_{2}-\mathrm{EP} 2$ signaling stabilizes the $\mathrm{Ccl} 2$ mRNA encoding MCP-1 by activating HuR (Fig. 7), resulting in the aneurysm formation (11). If macrophages in the adventitia come from the blood, endothelial cells should be activated. NF- $\mathrm{BB}$ was activated in endothelial cells of intracranial aneurysm walls at the early stage of intracranial aneurysm formation (Fig. 2), and our previous study also implicates EP2 signaling in this process (18). However, unexpectedly, the present study revealed that both NF- $\mathrm{KB}$ activation and EP2 signaling in endothelial cells were dispensable for intracranial aneurysm formation and macrophage-mediated inflammation in the lesion (Figs. 2 and 3). These findings indicate that signaling through other pathways is critically important for endothelial cell activation. Mechanotransduction mechanisms that sense hemodynamic force and convert it to intracellular signaling in endothelial cells are an active research field, and various mechanisms involving primary cilia, ion channels, integrin, plasma membrane fluidity, or actin cytoskeleton are proposed as components of the mechanosensing mechanisms (58). It will therefore be important to discover the mechanism that senses hemodynamic stress in endothelial cells that leads to subsequent macrophage infiltration.

\section{MATERIALS AND METHODS Experimental design}

For all animal studies, animals were randomized and investigators were blinded to group allocation during data collection. At least three independent samples were analyzed in all experiments.

\section{Study approval}

The animal experiments in this study complied with the National Institutes of Health Guide for the Care and Use of Laboratory Animals and were approved by the Institutional Animal Care and Use Committees 
of Kyoto University Graduate School of Medicine and RIKEN Kobe Branch. A series of patient-derived tissue samples from 16 unruptured and 20 ruptured intracranial aneurysms have been previously described $(20,55)$. These tissue samples were collected during microsurgical clipping of intracranial aneurysms at the Department of Neurosurgery, Helsinki University Central Hospital, Helsinki, Finland. We obtained informed consent from patients for their participation in the study after the nature and possible consequences were explained; the study was approved by the Operative Ethical review committee of Helsinki University Central Hospital (approval number 21/13/03/02/2013).

\section{Immunohistochemistry and immunofluorescence staining of human intracranial aneurysm tissue samples}

The detailed histopathology procedures performed on this set of samples have been described previously $(20,59)$. Briefly, for immunohistochemistry, frozen sections (10 $\mu \mathrm{m}$ thick) were fixed, blocked, and incubated with primary antibodies. Immune complexes were detected with either a Vectastain Elite ABC kit (Vector Laboratories) or an EnVision kit (DAKO) using 3,3'-diaminobenzidine as substrate. Sections were costained with Mayer's hematoxylin (Sigma-Aldrich) to visualize nuclei, and in the case of CD68 immunostaining, sections were also costained with Oil Red O. In negative controls, primary antibodies were either omitted or replaced with an irrelevant IgG1 (Serotec). Resected human tonsils and cadaver intracranial arteries served as positive controls. Images were captured using a Zeiss Axioplan 2 imaging microscope (Flokal Bv Micro-Optik) equipped with a ProgRes C7 USB digital camera (Jenoptik).

For double immunofluorescence staining, frozen sections were fixed, blocked with either 3\% normal goat or 3\% normal horse serum, and incubated with primary antibodies, followed by detection with Alexa 488-conjugated anti-mouse, Alexa 594-conjugated anti-sheep, or Alexa 594-conjugated anti-rabbit secondary antibodies (Molecular Probes). The sections were mounted using a Vectashield medium with DAPI (Vector Laboratories). An incubation omitting primary antibody was used as the negative control. Immunofluorescence images were captured with a 3DHISTECH Pannoramic 250 Flash II digital slide scanner or an LSM700 Zeiss confocal microscope system. Maximum intensity projections were generated from $\mathrm{z}$ sections using the ImageJ program (https://imagej.nih.gov/ij/index.html). Primary antibodies used were mouse monoclonal anti-human COX-2 antibody (DAKO), rabbit polyclonal anti-human EP2 antibody (LifeSpan Biosciences Inc.), mouse monoclonal anti-CD31 antibody (DAKO), mouse monoclonal anti-CD68 antibody (DAKO), and mouse monoclonal anti- $\alpha$-smooth muscle actin antibody (Sigma-Aldrich).

\section{Animals, rodent intracranial aneurysm models, and histological analysis of induced intracranial aneurysm}

Sprague-Dawley rats and C57BL/6NCrSlc mice were purchased from Japan SLC. Lyz2-Cre transgenic mice and Cdh5-Cre transgenic mice were purchased from The Jackson Laboratory. The Ptger2 flox mouse line, NF- $\kappa \mathrm{B}$ reporter mouse line, and $I \kappa B$ mutant transgenic mouse line were established in the present study and deposited to RIKEN, Japan (accession nos. CDB0939K, CDB0509T, and CDB0508T; www2. clst.riken.jp/arg/mutant\%20mice\%20list.html; www2.clst.riken.jp/arg/ TG\%20mutant\%20mice\%20list.html). Animals were maintained on a light/dark cycle of 14 hours/10 hours under specific pathogen-free conditions and had free access to chow and water.

Intracranial aneurysms were induced in 7-week-old male rats or mice by ligation of the left common carotid artery followed by induc- tion of systemic hypertension, which was achieved by salt overloading and ligation of the left renal artery. Both surgeries were conducted under general anesthesia induced by intraperitoneal injection of sodium pentobarbital (50 mg/kg). This procedure, which is referred to as "intracranial aneurysm induction" in this paper, is designed to increase hemodynamic stress on bifurcation site of intracranial arteries in the right, a contralateral side of the carotid ligation $(8,18)$. Immediately after intracranial aneurysm induction, animals were fed chow containing 8\% sodium chloride and 0.12\% 3-aminopropionitrile (\#A0408, Tokyo Chemical Industry), an inhibitor of lysyl oxidase, an enzyme that catalyzes the cross-linking of collagen and elastin. 3-Aminopropionitrile was used to facilitate intracranial aneurysm formation and progression by exacerbating arterial wall fragility. Intracranial aneurysm induction was assessed at the bifurcation of the right anterior cerebral artery (ACA) and the olfactory artery (OA) at the indicated times in rats and at 5 months in mice after induction, respectively. These sites were chosen because intracranial aneurysm formation was found to be consistent. Systolic, diastolic, and mean blood pressures were measured using the tail-cuff method (BP-98A, Softron) without any anesthesia. Systemic blood pressure measurements reported represent an average of at least three independent measurements from each animal. For histological analysis, animals were sacrificed after injection with a lethal dose of sodium pentobarbital. Animals were transcardially perfused with $4 \%$ paraformaldehyde, the ACA-OA bifurcation containing the intracranial aneurysm was then dissected from the circle of Willis, and $5-\mu \mathrm{m}$-thick serial frozen sections were prepared. Some of the sections were subjected to Elastica van Gieson staining to visualize the elastic lamina in the intracranial aneurysm walls. An aneurysm was defined as a lesion with disruption of the internal elastic lamina, as previously reported $(15,18)$. The size of the induced intracranial aneurysms was evaluated by a maximum transverse diameter as "Maximum" and a height as "Height" using Elastica van Gieson-stained sections.

\section{Generation of Ptger2 flox mouse line}

A Ptger 2 flox mouse was designed to delete exon 1 of the Ptger 2 gene, which encodes the first to sixth transmembrane domains, in the presence of Cre recombinase. Exon 1 of the Ptger2 gene was cloned using PCR amplification from a BAC clone (\#RP24-128I4 from BACPAC Resources) as template and ligated in the DT-ApA/conditional KO FW vector obtained from RIKEN (www2.clst.riken.jp/arg/cassette.html) using the Pme I sites. The targeting vector was then injected into embryonic stem (ES) cells, and after selection with neomycin and subsequent screening by PCR and Southern blot analyses, an ES cell line with the expected homologous recombination was established. F0 mice were then generated from ES clones and selected again by PCR and Southern blot analyses. After the F1 strain containing the Ptger2 floxed allele was selected by genotyping, the neomycin-resistant gene was deleted by crossing these mice with FLP (flippase) transgenic mice to establish the Ptger $2^{\text {flox/flox }}$ mouse strain (accession no. CDB0939K; www2.clst.riken.jp/arg/mutant\%20mice\%20list.html). Correct ablation of exon 1 of Ptger2 in the presence of Cre recombinase was confirmed by direct sequencing analysis of offspring obtained from crossing the Ptger $2^{\text {flox/flox }}$ line with the EIIa-Cre line (3130xl Genetic Analyzer from Life Technologies Corporation).

\section{Generation of cell type-specific Ptger2 knockout mice and intracranial aneurysm induction}

To generate macrophage- and endothelial cell-specific Ptger2-deficient mice, Ptger $2^{\text {flox/flox }}$ mice were crossed with Lyz2-Cre and Cdh5-Cre 
transgenic mice (The Jackson Laboratory), respectively. Genotyping by PCR was performed to detect the flox allele and the Cre gene using KOD Plus DNA polymerase (Toyobo). Primer sets used in this step were as follows: 5'-GCGGTCTGGCAGTAAAAACTATC-3' (forward) and 5'-GTGAAACAGCATTGCTGTCACTT-3' (reverse) for the detection of $\mathrm{Cre} ; 5^{\prime}$-CACGGTTCCATTTCTTGGATGG-3' (forward)

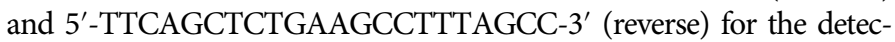
tion of the flox allele (wild-type allele, $243 \mathrm{bp}$; flox allele, $444 \mathrm{bp}$ ).

\section{Generation of an NF-кB reporter mouse line and induction of intracranial aneurysm}

Six tandem NF- $\kappa \mathrm{B}$ binding sites and a minimal promoter sequence were inserted into the promoterless pTimer-1 vector from Clontech Laboratories Inc. After confirming correct NF- $\kappa \mathrm{B}-$ regulated expression of Timer-1 protein in vitro [stimulation with TNF- $\alpha$ (100 ng/ml) for 24 hours] (fig. S3B), the vector was linearized and injected into $\mathrm{C} 57 \mathrm{BL} / 6$ fertilized eggs to generate transgenic mice. Offspring were screened by genotyping using PCR analysis to detect the Timer-1 gene using KOD Plus DNA polymerase (Toyobo) and the following primer sets: 5'-GACGGCTGCTTCATCTACAAGG-3' (forward) and 5' GTGATGTCCAGCTTGGTGTCC-3' (reverse) (product size, 263 bp). Regulated expression of Timer-1 protein under NF- $\kappa \mathrm{B}$-activating conditions in this NF- $\kappa \mathrm{B}$ reporter mouse line (accession no. CDB0508T; www2.clst.riken.jp/arg/TG\%20mutant\%20mice\%20list.html) was assessed in LPS-induced hepatitis (20 mg/kg, 4 hours) (fig. S3C) or DSSinduced colitis (fig. S3D).

Induction of intracranial aneurysms was performed in these different mouse lines, as described previously. At the indicated times after surgical induction, mice were sacrificed and 5- $\mu$ m-thick slices from the walls of the intracranial aneurysm were prepared as described previously. NF- $\kappa \mathrm{B}$ activation in intracranial aneurysm walls was examined by immunohistochemical detection of the Timer-1 protein with an antibody recognizing RFP (Rockland Immunochemicals Inc.). To evaluate the type of cells in which NF- $\kappa \mathrm{B}$ was activated, double immunostaining was performed using both an antibody that recognizes RFP and an antibody for each cell marker: for endothelial cells, a rat monoclonal antibody recognizing mouse CD31 (BD Biosciences); for smooth muscle cells, a mouse monoclonal antibody recognizing human $\alpha$-smooth muscle actin (Thermo Fisher Scientific); and for macrophages, a rat monoclonal antibody recognizing F4/80 (Abcam).

\section{Generation of a Ptger2-deficient NF-кB reporter mouse line} To examine the effects of Ptger 2 deficiency on NF- $\kappa \mathrm{B}$ activation in vivo, we generated a Ptger2-deficient NF- $\kappa \mathrm{B}$ reporter transgenic mouse line by crossing Ptger2-deficient mice with NF- $\kappa \mathrm{B}$ reporter mice. Offspring were screened by genotyping in PCR analysis to detect both the absence of the Ptger2 allele and the presence of Timer-1 gene using a KOD Plus DNA polymerase (Toyobo) and the primer sets described previously.

\section{Generation of conditional / $\kappa B$ mutant transgenic mouse line and intracranial aneurysm induction}

The mouse $I \kappa B \alpha$ gene was cloned by PCR using complementary DNA (cDNA) prepared from total RNA purified from mouse kidney, and its sequence was confirmed by sequencing. Site-directed mutations at two serine residues (S32A and S36A) in the $I \kappa B \alpha$ gene were generated using a KOD Plus Mutagenesis kit (Toyobo). Mutated $I \kappa B \alpha$ was then fused in-frame with the $m$ Cherry gene to allow monitoring of the I $\mathrm{B}$ mutant protein expression and then cloned into the pCCALL2 vector, which contains the loxP-stop-loxP sequence under the transcriptional control of the CAG promoter. After in vitro confirmation of the regulated expression of the mutated $\mathrm{I} \kappa \mathrm{B} \alpha$-mCherry fusion protein under the control of Cre recombinase, resistance to TNF- $\alpha$ (100 ng/ml, $20 \mathrm{~min})-$ induced degradation (fig. S8B), and also potent inhibitory capability of NF- $\kappa \mathrm{B}$ activation under stimulation with TNF- $\alpha$ (100 ng/ml, $20 \mathrm{~min})$ (fig. S8C), the vector harboring the mutated $I \kappa B \alpha-m$ Cherry construct was linearized and injected into C57BL/6 fertilized eggs. Proper integration of the transgene into the mouse genome was confirmed by genotyping and also by Southern blot analysis. From this, we obtained four independent lines of loxP-stop-loxP-I $\mathrm{K}$ mutant-mCherry conditional transgenic mice. The regulated expression of the mutated $\mathrm{I} \kappa \mathrm{B} \alpha-\mathrm{mCherry}$ fusion protein in the presence of Cre recombinase was assessed in these mice (accession no. CDB0509T; www2.clst.riken. jp/arg/TG\%20mutant\%20mice\%20list.html) by transfecting primary cultures of macrophages obtained from either the intraperitoneal cavity or the femoral muscle with a Cre recombinase expressing plasmid (48 hours) (fig. S8, D and E).

To establish endothelial cell- or macrophage-specific I $\mathrm{B}$ mutant$\mathrm{mCherry}$ fusion protein-expressing mice, loxP-stop-loxP-IКB mutant$m$ Cherry conditional transgenic mice (line \#12) were crossed with Cdh5-Cre or Lyz2-Cre transgenic mice line (The Jackson Laboratory), respectively. Offspring were then genotyped by PCR screening to detect the flox allele and the Cre gene using a KOD Plus DNA polymerase (Toyobo). The primer sets used were as follows: 5'-GCGGTCTGGCAGTAAAAACTATC-3' (forward) and 5'-GTGAAACAGCATTGCTGTCACTT-3' (reverse) to detect Cre; 5'-GCCAAGCTGAAGGTGACCAAGG-3' (forward) and 5'-GCTTCAGCCTCTGCTTGATCTCG-3' (reverse) to detect the $I \kappa B$ mutant-mCherry sequence (product size, $370 \mathrm{bp}$ ). Mice for each genotype were obtained in the expected Mendelian ratios.

\section{Southern blot analysis}

Southern blot analysis was performed to confirm the correct recombination of the genome of genetically modified mice (figs. S5B and S7C). Briefly, mouse genomic DNA was purified from the kidney of each mouse line and digested with restriction enzymes Swa I, Sca I, Bgl II, or Eco RV for at least 48 hours until complete digestion was confirmed by electrophoresis. Digested DNA $(50 \mu \mathrm{g})$ was then separated by electrophoresis on a $0.5 \%$ agarose gel, transferred to a nylon membrane (Hybond-N, GE Healthcare), and hybridized with digoxigenin-labeled probes generated by in vitro transcription (DIG High Prime DNA Labeling and Detection Starter Kit II, Roche Diagnostics). Probe locations within the genome are indicated in the map of each mouse line. Signal was detected using a chemiluminescence reagent (CDP-Star, Roche).

\section{RNA extraction from in vivo samples and quantitative RT-PCR analysis}

Five months after intracranial aneurysm induction, animals were sacrificed. Total RNA was purified from the anterior portion of the circle of Willis and reverse-transcribed into cDNA using an RNeasy Fibrous Tissue Mini kit (Qiagen) and a High-Capacity cDNA Reverse Transcription kit (Life Technologies) according to the manufacturer's instructions. Quantitative RT-PCR analysis was then performed with a SYBR Premix Ex Taq II kit (Takara) and a Real-Time System CFX96 (Bio-Rad). Actb (which encodes $\beta$-actin) was used as an internal control [primer sets: 5' -ACGACCAGAGGCATACAGGGA-3' (forward) and $5^{\prime}$-CCCTAAGGCCAACCGTGAAA-3' (reverse)]. For quantification, 
the second derivative maximum method was used for crossing point determination, and the value of each gene expression was normalized to $A c t b$ expression. Primer sets used in the RT-PCR were as follows: 5'-GTCTCTGCCGCCCTTCTGTG-3' (forward) and 5'-AGGTGACTGGGGCATTGATTG-3' (reverse) for mouse Ccl2; 5' CTTCTGCAGTCCAGGTTCAATGG-3' (forward) and 5'-AAGACTTGCCAGGCTGAACTTCG-3' (reverse) for mouse Ptgs2; and $5^{\prime}$-CCAAGACAAGGAAGGTTAGGC-3' (forward) and 5'-GGAAGTATTTGGCTCCATTCC-3' (reverse) for rat Spl.

\section{Immunohistochemistry of tissue specimens}

Immunohistochemistry was performed as previously described (18). Briefly, at 1,2, 4, 8, 20,27, and 32 weeks in mice or at 3, 4, or 6 weeks in rats after intracranial aneurysm induction, animals were sacrificed and perfused transcardially with $4 \%$ paraformaldehyde. The right ACA-OA bifurcation was then dissected from the circle of Willis, and $5-\mu \mathrm{m}$-thick tissue sections were prepared. In some analyses, tissues from other organs, namely, spleen, lung, aorta, and kidney, were dissected and similar tissue slices were made. After blocking the tissue slices with 5\% donkey serum (Jackson ImmunoResearch), primary antibodies diluted in phosphate-buffered saline were incubated with the slices, followed by incubation with secondary antibodies conjugated with a fluorescent dye (Jackson ImmunoResearch). Fluorescent images were acquired using a CTR6500 confocal fluorescence microscope (Leica Microsystems). Cells positive for F4/80 in mice or CD68 in rats by immunohistochemistry were defined as macrophages. The number of infiltrated macrophages found within a $100-\mu \mathrm{m}$ square around the dome of the induced aneurysms was calculated.

Primary antibodies used were as follows: a rat monoclonal antibody recognizing mouse CD31 (BD Biosciences), a mouse monoclonal antibody recognizing human $\alpha$-smooth muscle actin (Thermo Fisher Scientific), a rat monoclonal antibody recognizing F4/80 (Abcam), a mouse monoclonal antibody recognizing CD68 (Abcam), a rabbit polyclonal antibody recognizing RFP (Rockland Immunochemicals), a rabbit monoclonal antibody recognizing the phosphorylated form of NF- $\kappa$ B p65 (Ser ${ }^{536}$ ) (Cell Signaling Technology), a rabbit polyclonal antibody recognizing Cre (Merck Millipore), a goat polyclonal antibody recognizing MCP-1 (Santa Cruz Biotechnology), and a rabbit polyclonal antibody recognizing COX-2 (Cayman Chemical).

\section{DSS-induced colitis in mice}

Experimental colitis was induced in mice by oral administration of DSS (molecular weight, 36,000 to 50,000; MP Biomedicals LLC). DSS was given to mice in drinking water (3\%) for 8 days followed by 3-day administration with normal drinking water. Eleven days after the start of DSS treatment, mice were sacrificed and the colon was dissected for analysis.

\section{EP2 antagonist treatment}

PF-04418948, 1-(4-fluorobenzoyl)-3-\{[(6-methoxy-2-naphthyl)oxy]methyl $\}$ azetidine-3-carboxylic acid, a selective EP2 antagonist with a $K_{\mathrm{i}}$ value of $16 \mathrm{nM}$ for human EP2 (41), was synthesized at KNC Laboratories Co. Ltd. The purity of synthesized PF- 04418948 was $99.1 \%$ by highperformance liquid chromatography. PF-04418948 was orally administered to rats by mixing it into chow to provide the doses indicated $(1,3,10$, and $50 \mathrm{mg} / \mathrm{kg})$ for 3 weeks. In some experiments, the intracranial aneurysm was first induced in rats for 1 week after surgical manipulation, and then, PF-04418948 (50 mg/kg) was administered daily for an additional 5 weeks.

\section{Microarray analysis}

Three weeks after intracranial aneurysm induction, rats were sacrificed. Total RNA was purified from the right anterior portion of the circle of Willis using an RNeasy Fibrous Tissue Mini kit (Qiagen). Purified RNA was then labeled with the fluorescent dye Cy3 using a Low Input Quick Amp Labeling Kit, One-Color (Agilent Technologies) and hybridized to array plates (SuperPrint G3 rat GE 8X60K Microarray, Agilent Technologies) using a Gene Expression Hybridization kit (Agilent Technologies). After washing the hybridized array, signals were detected using a microarray scanner (Agilent Technologies). Data were analyzed using the GeneSpring software (Agilent Technologies). The fold changes in expressions of each gene in the intracranial aneurysm lesions were calculated as a ratio compared to that in the ring of Willis from sham-operated rats.

\section{Compounds used in in vitro experiments}

Cultured cells were treated with the following compounds, as indicated in Materials and Methods, the figure legends, and figures: LPS (Sigma-Aldrich), EP2 agonist (ONO-AE1-259, gift from Ono Pharmaceutical Co. Ltd.), TNF- $\alpha$ (R\&D Systems), IFN- $\gamma$ (R\&D Systems), H-89 (Merck KGaA), SB202190 (Merck KGaA), SB239063 (Merck KGaA), mithramycin A (Santa Cruz Biotechnology), leptomycin B

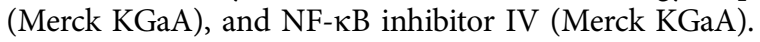

\section{Generation of a HEK293 cell line stably expressing EP2}

The open reading frame (ORF) of mouse Ptger 2 was obtained by PCR using PrimeSTAR Max DNA polymerase (Takara), and its sequence was confirmed. The ORF of Ptger2 was then cloned into the pLenti6.3 vector containing the blasticidin resistance gene as a selection marker (Life Technologies). Subsequently, Lenti-X cells were transfected with the pLenti6.3-murine Ptger2 plasmid and packaging plasmids (Clontech) to generate lentiviral particles harboring Ptger2, which were used to infect HEK293 cells. The pLenti6.4-GFP plasmid was used as a control to monitor infection efficiency. Finally, infected HEK293 cells were selected for resistance to blasticidin for 120 hours to establish the HEK293 cell line stably expressing EP2 (HEK293-EP2) (fig. S12A). The concentration of blasticidin used was $6 \mu \mathrm{g} / \mathrm{ml}$ because this dose killed all noninfected cells. To functionally validate the established HEK293-EP2 cell line, the cells were stimulated with the selective EP2 agonist ONO-AE1-259 [median effective concentration $\left(\mathrm{EC}_{50}\right), 0.003 \mu \mathrm{M}$ ] $(0.00005,0.005$, and $0.5 \mu \mathrm{M}$ for $15 \mathrm{~min})(28)$, and dose-dependent increases in cAMP were measured using a Cyclic AMP EIA kit (Cayman Chemical) according to the manufacturer's instructions (fig. S12C).

In RT-PCR experiments, cells were stimulated with ONO-AE1-259 $(0.5 \mu \mathrm{M}), \mathrm{TNF}-\alpha(1 \mathrm{ng} / \mathrm{ml})$, or both agents in combination for 1,2 , or 3 hours, and total RNA was isolated for further analysis (Fig. 5A and figs. S13D and S15A). To examine the role of posttranscriptional modifications on Ccl2 or Ptgs 2 mRNA induction after EP2 stimulation, actinomycin D $(20 \mu \mathrm{g} / \mathrm{ml})$ and $0.5 \mu \mathrm{M}$ ONO-AE1-259 were added to cells prestimulated with TNF- $\alpha(100 \mathrm{ng} / \mathrm{ml})$ for 2 hours, and the decrease in $\mathrm{Ccl} 2$ or Ptgs 2 mRNA levels was examined by RT-PCR (Fig. 5B and fig. S17A).

\section{Generation of an NF-кB reporter HEK293 cell line}

The $\kappa B-G F P$ cassette was transduced into HEK293 cells using the lentivirus system (Cignal Lenti Reporter Assay, Qiagen) according to the manufacturer's instructions, and infected cells were cloned by selection in puromycin $(1600 \mathrm{ng} / \mathrm{ml})$ for 2 weeks. The effect of the EP2 agonist ONO-AE1-259 (0.5 $\mathrm{M}, 24$ hours) on NF- $\kappa \mathrm{B}$ activation 
was examined by assessing GFP expression using TNF- $\alpha$ stimulation (100 ng/ml, 24 hours) as a positive control (fig. S13A).

\section{Preparation of macrophage primary cultures}

Primary macrophages were obtained from the intraperitoneal cavity of rats that had been injected intraperitoneally with a $3 \%$ thioglycollate broth. After 4 days, the peritoneal cavity was washed with ice-cold RPMI 1640 medium supplemented with $10 \%$ fetal bovine serum and cells resident in the peritoneal cavity were collected. After washing, the cells were seeded in culture dishes and indomethacin $(100 \mu \mathrm{M}$, 2 hours) was added to inhibit endogenous $\mathrm{PGE}_{2}$ production before cells were used in in vitro experiments. Cells were then stimulated with ONO-AE1-259 (0.5 $\mu \mathrm{M}, 2$ hours) or TNF- $\alpha$ stimulation $(1 \mathrm{ng} / \mathrm{ml}$, 2 hours) or a combination of both agents (Fig. 4D). In some experiments, macrophages were treated with NF- $\kappa \mathrm{B}$ inhibitor IV $(50 \mu \mathrm{M})$ and ONO-AE1-259 (0.5 $\mu \mathrm{M}, 1$ hour) with or without TNF- $\alpha$ stimulation (5 ng/ml, 1 hour) (fig. S14D).

\section{Quantitative RT-PCR analysis in cultured cells}

RNA purification from cultured cells and reverse transcription of purified RNA were performed using an RNeasy Mini kit (Qiagen) and a High-Capacity cDNA Reverse Transcription kit (Life Technologies Corporation), according to the manufacturers' instructions. For quantification of gene expression, RT-PCR was performed on Real-Time System CFX96 (Bio-Rad) using SYBR Premix Ex Taq II (Takara). Actb was used as the internal control. For quantitation, the second derivative maximum method was used for crossing point determination.

Primers used were as follows: $5^{\prime}$-CATACTCCTGCTTGCTGATCC$3^{\prime}$ (forward) and 5'-GATGCAGAAGGAGATCACTGC-3' (reverse) for human $A c t b$; 5'-ACACCCTCTATCACTGGCATCC-3' (forward) and 5'-AACATTCCTACCACCAGCAACC-3' (reverse) for human Ptgs2; 5'-AGCTTCTTTGGGACACTTGC-3' (forward) and 5'-ATAGCAGCCACCTTCATTCC-3' (reverse) for human Ccl2; 5'-CTGTTGCCCATCAACGGTCTG-3' (forward) and 5' $5^{\prime}$ AATGGCAGCAATGGCAGTGAG-3' (reverse) for human Sp1; 5'-ACATGGTCATGGTCAAAGAGG-3' (forward) and 5'-TTGTAAGTCACCGCCAGTACC-3' (reverse) for human Elavl1; 5'-GGGCAGTAATCTCCTTCTGCATCC-3'

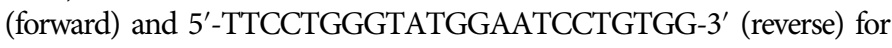
rat $A c t b ; 5^{\prime}$-GATGTGGATGCTTTCAAGTCC-3' (forward) and 5' CCTGTGAGCAGTCAGAGAAGG-3' (reverse) for rat Fos; 5' -ACTAGCAGGTCGTCATCATCC-3' (forward) and 5'-CATCTCGACAAGAGCTTCAGG-3' (reverse) for rat $I l 1 b$; $5^{\prime}$-TCCTTTCCAGGTCAGTTAGCC-3' (forward) and 5'-GCCCAGACAGAAGTCATAGCC-3' (reverse) for rat $\mathrm{Cxcl} 2$; 5'-CCGGACTTGTGAAGTAGGGAAGG-3' (forward) and 5'-CCACCAGGAACGAAAGTCAACTCC-3' (reverse) for rat Il6; 5'-TCAGTATGAGCCTGCTGGTTTGG-3' (forward) and 5'-CCGGGTCTGATGATGTATGCTACC-3' (reverse) for rat Ptgs2; and 5'-GTCAGAGTCTTGTGCCTTTGG-3' (forward) and 5'-CCAAGCTGAACTTGAGTGAGG-3' (reverse) for rat Nos2.

\section{Luciferase assays}

A luciferase assay was used to examine the promoter activity of Ptgs2, the transcriptional activity of Sp1 and NF- $\kappa B$, or the effect of the $3^{\prime}$ UTR of Ccl 2 mRNA on mRNA stabilization. Luciferase activity was measured using the Dual-Luciferase Reporter Assay System and the GloMax Microplate Luminometer (Promega) according to the manufacturer's instructions. Constructs used in these experiments were generated by ligating a PCR-amplified fragment of the gene promoter of interest into the multicloning site of the pGL4.17 vector harboring the
ORF of firefly-luciferase (Promega). The Sp1 reporter construct was made by ligating, in tandem, three consensus Sp1 binding sites along with the minimum promoter upstream of the firefly-luciferase ORF. To establish the NF- $\mathrm{BB}$ reporter in HEK293 cells, the NF- $\kappa \mathrm{B}-$ fireflyluciferase cassette and the control Renilla-luciferase cassette were transduced into HEK293 cells using the lentivirus system (Cignal Lenti Reporter Assay) according to the manufacturer's instructions, and infected clones were selected using puromycin (1600 ng/ml, 2 weeks). To examine mRNA stabilization by the RNA binding protein $\mathrm{HuR}$, the 3'UTR of human CCL2 mRNA was cloned [using the following primer set: $5^{\prime}$-ACACTCACTCCACAACCCAAG-3' (forward) and $5^{\prime}$-TTTGGTGTAATAGTTACAAAA-3' (reverse)] and ligated into the $3^{\prime}$ end of the firefly-luciferase ORF driven under the transcriptional control of the PGK (phosphoglycerate kinase) promoter (Promega). Site-directed mutagenesis or construct deletion was achieved using an inverse PCR-based method using a KOD Plus Mutagenesis kit (Toyobo). Some mutants were constructed by restriction enzyme digestion of the original construct and self-ligation. In these experiments, a Renilla-luciferase construct driven by the TK (thymidine kinase) promoter (Promega) cotransfected with the firefly-luciferase construct was used as the internal control.

In the luciferase assay, to examine the transcriptional regulation of Ptgs 2 by EP2 and TNF- $\alpha$ signaling, cells transfected with a construct harboring the Ptgs 2 promoter sequence or its mutants were stimulated with ONO-AE1-259 (0.005 or $0.5 \mu \mathrm{M}, 6$ hours) or TNF- $\alpha(1 \mathrm{ng} / \mathrm{ml}$, 6 hours) (Fig. 4E and fig. S15, B and C). To examine the transcriptional activity of Sp1, HEK293-EP2 cells were transfected with the Sp1 reporter firefly-luciferase plasmid and control Renilla-luciferase plasmid and stimulated with ONO-AE1-259 $(0.5 \mu \mathrm{M})$ or TNF- $\alpha(1 \mathrm{ng} / \mathrm{ml})$ or in combination for 2, 6, and 24 hours (fig. S16B). To further examine the effect of Sp1 on Ptgs2 promoter activity, cells treated with the Sp1 inhibitor mithramycin $(0.1,0.5$, or $1 \mu \mathrm{M})$ or transfected with siRNA to deplete $S p 1$ or an Sp1-overexpressing plasmid were stimulated with ONO-AE1-259 $(0.5 \mu \mathrm{M})$ or TNF- $\alpha(1 \mathrm{ng} / \mathrm{ml})$ or in combination for 6 hours (fig. S16, B to E). To examine the effect of EP2 signaling on stabilization of Ccl2 mRNA, HEK293-EP2 cells transfected with the luciferase-Ccl2-3'UTR construct or its mutants were treated with $0.5 \mu \mathrm{M}$ ONO-AE1-259 alone for 12 hours or in combination with the p38 inhibitor SB202190 $(0.1$ or $1 \mu \mathrm{M})$, SB239063 (1 or $10 \mu \mathrm{M})$, or leptomycin $\mathrm{B}(0.4,4$ or $40 \mathrm{nM})$ and analyzed by luciferase assay (Fig. 5, C to E, and fig. S17D). To examine the effect of NF- $\kappa B$ inhibitor IV on NF- $\kappa B$ transcriptional activity, NF- $\kappa \mathrm{B}$ reporter HEK293 cells were treated with each dose of NF- $\mathrm{KB}$ inhibitor IV in combination with TNF- $\alpha$ stimulation (10 ng/ml, 6 hours) (fig. S14C).

\section{Immunohistochemistry and Western blot analysis using in vitro samples}

For immunohistochemistry, cells were cultured on chamber slides and, in some experiments, stimulated with compounds [ONO-AE1-259: $0.5 \mu \mathrm{M}, 24$ hours; TNF- $\alpha$ : $100 \mathrm{ng} / \mathrm{ml}, 20 \mathrm{~min}$ (fig. S8B) or 24 hours (fig. S3B)]. Cells were then fixed with $4 \%$ paraformaldehyde. After blocking with $5 \%$ donkey serum (Jackson ImmunoResearch), samples were incubated with primary antibodies, followed by incubation with secondary antibodies conjugated with a fluorescent dye (Jackson ImmunoResearch). Fluorescent images were acquired on a confocal fluorescence microscope system (CTR6500, Leica Microsystems).

For Western blot analysis, whole-cell lysates were prepared from treated cells (ONO-AE1-259: 0.00005, 0.005, or $0.5 \mu \mathrm{M}$ for $20 \mathrm{~min}$ and $0.5 \mu \mathrm{M}$ for $20,60,120$, or $240 \mathrm{~min}$; db-cAMP: 30 or $100 \mu \mathrm{M}$ for 
$20 \mathrm{~min}$; H-89: 1 or $10 \mu \mathrm{M}$ for $20 \mathrm{~min}$; TNF- $\alpha$ : $1 \mathrm{ng} / \mathrm{ml}$ for $20 \mathrm{~min}$; LPS: $1 \mu \mathrm{g} / \mathrm{ml}$ for $30 \mathrm{~min}$ ) (Fig. 4, B and C, and figs. S13B, S14A, and S18B) by adding RIPA solution supplemented with proteinase and phosphatase inhibitors (Sigma-Aldrich). In some experiments, cytoplasmic and nuclear fractions were prepared separately using a Nuclear Extraction kit (Active Motif), from HEK293-EP2 cells treated with $0.5 \mu \mathrm{M}$ ONO-AE1-259 for 8 hours (fig. S17C) or RAW264.7 cells (purchased from Public Health England) stimulated with LPS $(1 \mu \mathrm{g} / \mathrm{ml}, 20 \mathrm{~min})$ in the presence or absence of NF- $\kappa \mathrm{B}$ inhibitor IV (50 $\mu \mathrm{M})$ (fig. S14B), and nuclear and cytosolic extracts were prepared according to the manufacturer's instructions. After SDS-polyacrylamide gel electrophoresis of the nuclear and cytoplasmic fractions $(20 \mu \mathrm{g})$, proteins were transferred to a polyvinylidene difluoride membrane (Hybond-P, GE Healthcare) and blocked with ECL Plus blocking agent (GE Healthcare). The blotted membranes were then incubated with primary antibodies followed by incubation with horseradish peroxidase-conjugated IgG (GE Healthcare). Finally, signals were detected using chemiluminescence (ECL Prime Western Blotting Detection System, GE Healthcare). $\alpha$ Tubulin (total cell lysate and cytoplasm) or lamin B (nuclear) served as an internal control.

Primary antibodies used were as follows: a rabbit monoclonal antibody recognizing p65 (Cell Signaling Technology), a rabbit monoclonal antibody recognizing a phosphorylated form of NF- $\kappa \mathrm{B}$ p 65 $\left(\operatorname{Ser}^{536}\right.$ ) (Cell Signaling Technology), a rabbit monoclonal antibody recognizing the phosphorylated form of NF- $\kappa$ B p105 $\left(\mathrm{Ser}^{337}\right.$ ) (Cell Signaling Technology), a mouse monoclonal antibody recognizing IкB $\alpha$ (Cell Signaling Technology), a rabbit monoclonal antibody recognizing p38 (Cell Signaling Technology), a rabbit monoclonal antibody recognizing the phosphorylated form of p38 $\left(\mathrm{Thr}^{180 / 182}\right.$ ) (Cell Signaling Technology), a rabbit monoclonal antibody recognizing TBP (TATAbinding protein) (Cell Signaling Technology), a mouse monoclonal antibody recognizing $\alpha$-tubulin (Sigma-Aldrich), a rabbit polyclonal antibody recognizing EP2 (Cayman Chemical), a goat polyclonal antibody recognizing lamin B (Santa Cruz Biotechnology), a mouse monoclonal antibody recognizing HuR (Santa Cruz Biotechnology), a rabbit polyclonal antibody recognizing RFP (Rockland Immunochemicals), a rat monoclonal antibody recognizing GFP (Nacalai Tesque Inc.), and a rabbit polyclonal antibody recognizing Sp1 (Merck Millipore)

\section{Transfections}

All plasmid transfections in cultured cells were performed using a liposome-based method (FuGENE, Promega). Cells were harvested and analyzed at least 24 hours after transfection.

\section{PCR array analysis}

PCR array analysis was performed according to the manufacturer's instructions (SABioscience, Qiagen) (Fig. 4D). Briefly, total RNA from primary cultures of intraperitoneal macrophages with TNF- $\alpha$ alone ( $5 \mathrm{ng} / \mathrm{ml}, 1$ hour) or in combination with ONO-AE1-259 $(0.5 \mu \mathrm{M})$ was purified using an RNeasy Plus Mini kit and subsequently reversetranscribed using an $\mathrm{RT}^{2}$ First Strand kit (Qiagen). PCRs were performed using $\mathrm{RT}^{2}$ qPCR Master Mix on Real-Time System CFX96 (Bio-Rad) according to the manufacturer's instructions. Finally, the relative expression of each gene was calculated and compared using the five internal controls supplied by the manufacturer.

\section{RNAi in cultured cells}

Cultured cells were transfected with commercially available predesigned Stealth siRNAs (Sp1, $10 \mathrm{nM}$; Elavl1, $30 \mathrm{nM}$ ) or a scrambled
siRNA using Lipofectamine RNAi MAX (Life Technologies) and cultured for an additional 72 hours to achieve sufficient knockdown (Fig. 5D and figs. S16D and S17B). The efficiencies of target mRNA and protein depletions were confirmed by quantitative RT-PCR analysis and Western blotting, respectively, using three independent biological replicates.

\section{Cloning and overexpression of Sp1}

The ORF of the human Sp1 gene (NM_138473.2) was prepared by PCR from cDNA prepared from HEK293 cells using the primers 5'-GTTCGCTTGCCTCGTCAG-3' (forward) and 5'-AATGCTTCATGGCTCTCTCTTC-3' (reverse) and cloned into the pcDNA3 vector.

\section{ChIP and Re-ChIP assay}

ChIP and Re-ChIP assays were performed according to the manufacturer's instructions using an EZ-Magna ChIP kit (Merck Millipore) and a Re-ChIP-IT kit (Active Motif). Cells were stimulated with either TNF- $\alpha(1 \mathrm{ng} / \mathrm{ml})$ or $0.5 \mu \mathrm{M}$ ONO-AE1-259 or both for $90 \mathrm{~min}$ and subjected to ChIP or Re-ChIP assays (Fig. 4, F and G). Antibodies used in these experiments were a mouse monoclonal antibody recognizing p65 (Merck Millipore) and a rabbit polyclonal antibody recognizing Sp1 (Merck Millipore). Primer sets used to detect the immunoprecipitated promoter fragments were as follows: 5'-AAAGACATCTGGCGGAAACC-3' (forward) and 5'-AAATCGGAAACCCAGGAAGC-3' (reverse) for the proximal NF- $\mathrm{KB}$ site; $5^{\prime}$-AAGACGTACAGACCAGACACG-3' (forward) and 5'-CAGAAGGACACTTGGCTTCC-3' (reverse) for the distal NF- $\mathrm{KB}$ site; and $5^{\prime}$-TTACCTTTCCCGCCTCTCTTTCC-3' (forward) and 5'-GTGACGACGCTTAATAGGCTG-3' (reverse) for the $\mathrm{Sp} 1$ site in the Ptgs 2 promoter.

\section{DNA binding activity of transcription factors}

The DNA binding activities of each NF- $\mathrm{KB}$ subunit (p50, p65, and c-Rel) and Sp1 were assessed in cultured cells (ONO-AE1-259: 0.5 $\mu \mathrm{M}$, $20 \mathrm{~min}$; TNF- $\alpha$ : $100 \mathrm{ng} / \mathrm{ml}, 20 \mathrm{~min}$ ) (Fig. 4A and figs. S13C and S16A) or after intracranial artery aneurysm induction (1 month) (fig. S16F) according to the manufacturer's instructions.

\section{Detection of MAPK phosphorylation by Proteome Profiler Array}

HEK293-EP2 cells were stimulated with ONO-AE1-259 (0.5 $\mu \mathrm{M}$, $20 \mathrm{~min}$ ), and whole-cell lysates were prepared (fig. S18A). Phosphorylation of each MAPK member was then detected in lysates $(300 \mu \mathrm{g})$ using a Proteome Profiler Human Phospho-MAPK Array kit (ARY002 from R\&D Systems) according to the manufacturer's instructions.

\section{RNA immunoprecipitation analysis}

RNA immunoprecipitation analyses were performed using a Magna RIP RNA-Binding Protein Immunoprecipitation kit (Merck Millipore) according to the manufacturer's instructions (Fig. 5F). Briefly, HEK293EP2 cells were stimulated with ONO-AE1-259 (0.5 $\mu \mathrm{M}, 8$ hours), and whole-cell lysates were prepared. RNA bound with HuR was then immunoprecipitated using a mouse monoclonal anti-HuR antibody (Santa Cruz Biotechnology) or normal mouse IgG as a negative control. RNA was purified from the immunoprecipitate and then reversetranscribed into cDNA. Finally, enrichment of the 3'UTR of $\mathrm{Ccl} 2$ was assessed using quantitative RT-PCR. The sequences of primers used for detecting $3^{\prime} \mathrm{UTR}$ of $\mathrm{Ccl} 2 \mathrm{mRNA}$ were $5^{\prime}$-AGCAATTTCCCCAAGTCTCTG-3' (forward) and 5'-ACCCAAGAATCTGCAGCTAAC-3' (reverse). 


\section{Statistics}

Data were shown as means \pm SEM. Differences between the two groups were examined using the nonparametric Mann-Whitney test. Statistical comparisons between more than two groups were conducted using Kruskal-Wallis test followed by the post hoc Steel test. The incidence of intracranial aneurysm across groups was analyzed using Fisher's exact test. Correlations between continuous and ordinal variables were examined using Spearman's rank correlation test. Nominal data were statistically compared using Fisher's exact test or the $\chi^{2}$ test, as appropriate.

\section{SUPPLEMENTARY MATERIALS}

www.sciencesignaling.org/cgi/content/full/10/465/eaah6037/DC1

Fig. S1. Scoring of COX-2 and EP2 abundance in human intracranial aneurysm lesions.

Fig. S2. Immunostaining for EP2, CD31, and/or $\alpha$-smooth muscle actin in human intracranial aneurysm lesions.

Fig. S3. Verification of the NF-kB reporter construct.

Fig. S4. NF- $\kappa B$ activation in intracranial arterial wall during intracranial aneurysm development assessed in NF- $\mathrm{KB}$ reporter mice.

Fig. S5. Generation of the Ptger $2^{\text {flox/flox }}$ mouse line.

Fig. S6. Effect of macrophage-specific deletion of Ptger2 on MCP-1 and COX-2

immunofluorescence in intracranial aneurysm lesions.

Fig. S7. Generation of the IKB mutant transgenic mouse line.

Fig. S8. Cre-dependent expression of the I $\mathrm{KB}$ mutant and suppression of NF- $\mathrm{KB}$ activation.

Fig. S9. Generation of transgenic mouse lines expressing a macrophage-specific $І \kappa B$ mutantmCherry fusion protein.

Fig. S10. Generation of transgenic mouse lines expressing $1 \kappa B$ mutant-mCherry fusion protein in an endothelial cell-specific fashion.

Fig. S11. Effects of macrophage- and endothelial cell-specific expression of the IкB mutant protein on systemic blood pressure and macrophage recruitment after intracranial aneurysm induction.

Fig. S12. Generation of the HEK293-EP2 cell line.

Fig. S13. NF- $K B$ activation in response to EP2 signaling in HEK293-EP2 cells.

Fig. S14. Involvement of NF- $\kappa B$ activation in amplifying the effect of EP2 on TNF- $\alpha$-induced gene expression.

Fig. S15. Analysis of the Ptgs2 gene promoter for $\kappa B$ sites responsive to EP2 and TNF- $\alpha$ signaling.

Fig. S16. Activation of Sp1 by TNF- $\alpha$ signaling in HEK293-EP2 cells.

Fig. S17. EP2-induced HuR translocation.

Fig. S18. Activation of the MAPK p38 by EP2 signaling in HEK293-EP2 cells.

Fig. S19. Effects of PF-04418948 on systemic blood pressure and thinning of media in intracranial aneurysm walls.

Table S1. Gene expression analysis of rat intracranial aneurysm lesions.

Data file S1. Full gene list from microarray analysis of rat intracranial aneurysm lesions.

\section{REFERENCES AND NOTES}

1. J. van Gijn, R. S. Kerr, G. J. E. Rinkel, Subarachnoid haemorrhage. Lancet 369, 306-318 (2007).

2. D. O. Wiebers, D. G. Piepgras, R. D. Brown Jr., I. Meissner, J. Torner, N. F. Kassell, J. P. Whisnant, J. Huston III, D. A. Nichols, Unruptured aneurysms. J. Neurosurg. 96, 50-51 (2002).

3. G. J. E. Rinkel, M. Djibuti, A. Algra, J. van Gijn, Prevalence and risk of rupture of intracranial aneurysms: A systematic review. Stroke 29, 251-256 (1998).

4. M. Kotowski, O. Naggara, T. E. Darsaut, S. Nolet, G. Gevry, E. Kouznetsov, J. Raymond, Safety and occlusion rates of surgical treatment of unruptured intracranial aneurysms: A systematic review and meta-analysis of the literature from 1990 to 2011. J. Neurol. Neurosurg. Psychiatry 84, 42-48 (2013).

5. J. P. Greving, M. J. H. Wermer, R. D. Brown Jr., A. Morita, S. Juvela, M. Yonekura, T. Ishibashi, J. C. Torner, T. Nakayama, G. J. E. Rinkel, A. Algra, Development of the PHASES score for prediction of risk of rupture of intracranial aneurysms: A pooled analysis of six prospective cohort studies. Lancet Neurol. 13, 59-66 (2014).

6. UCAS Japan Investigators, The natural course of unruptured cerebral aneurysms in a Japanese cohort. N. Engl. J. Med. 366, 2474-2482 (2012).

7. T. Aoki, M. Nishimura, Targeting chronic inflammation in cerebral aneurysms: Focusing on NF- $\mathrm{KB}$ as a putative target of medical therapy. Expert Opin. Ther. Targets 14, 265-273 (2010).
8. T. Aoki, M. Nishimura, The development and the use of experimental animal models to study the underlying mechanisms of CA formation. J. Biomed. Biotechnol. 2011, 535921 (2011).

9. T. Aoki, S. Narumiya, Prostaglandins and chronic inflammation. Trends Pharmacol. Sci. 33, 304-311 (2012).

10. J. M. Dolan, J. Kolega, H. Meng, High wall shear stress and spatial gradients in vascular pathology: A review. Ann. Biomed. Eng. 41, 1411-1427 (2013).

11. A. S. Turjman, F. Turjman, E. R. Edelman, Role of fluid dynamics and inflammation in intracranial aneurysm formation. Circulation 129, 373-382 (2014).

12. E. Metaxa, M. Tremmel, S. K. Natarajan, J. Xiang, R. A. Paluch, M. Mandelbaum, A. H. Siddiqui, J. Kolega, J. Mocco, H. Meng, Characterization of critical hemodynamics contributing to aneurysmal remodeling at the basilar terminus in a rabbit model. Stroke 41, 1774-1782 (2010).

13. M. Fukuda, T. Aoki, Molecular basis for intracranial aneurysm formation. Acta Neurochir. Suppl. 120, 13-15 (2015).

14. J. Cebral, E. Ollikainen, B. J. Chung, F. Mut, V. Sippola, B. R. Jahromi, R. Tulamo, J. Hernesniemi, M. Niemelä, A. Robertson, J. Frösen, Flow conditions in the intracranial aneurysm lumen are associated with inflammation and degenerative changes of the aneurysm wall. AJNR Am. J. Neuroradiol. 38, 119-126 (2016).

15. T. Aoki, H. Kataoka, M. Shimamura, H. Nakagami, K. Wakayama, T. Moriwaki, R. Ishibashi, K. Nozaki, R. Morishita, N. Hashimoto, NF-KB is a key mediator of cerebral aneurysm formation. Circulation 116, 2830-2840 (2007).

16. T. Aoki, H. Kataoka, R. Ishibashi, K. Nozaki, N. Hashimoto, Simvastatin suppresses the progression of experimentally induced cerebral aneurysms in rats. Stroke 39, 1276-1285 (2008).

17. T. Hirata, S. Narumiya, Prostanoid receptors. Chem. Rev. 111, 6209-6230 (2011).

18. T. Aoki, M. Nishimura, T. Matsuoka, K. Yamamoto, T. Furuyashiki, H. Kataoka, S. Kitaoka, R. Ishibashi, A. Ishibazawa, S. Miyamoto, R. Morishita, J. Ando, N. Hashimoto, K. Nozaki, S. Narumiya, $\mathrm{PGE}_{2}-\mathrm{EP} 2$ signalling in endothelium is activated by haemodynamic stress and induces cerebral aneurysm through an amplifying loop via NF-кB. Br. J. Pharmacol. 163, 1237-1249 (2011).

19. T. Aoki, H. Kataoka, R. Ishibashi, K. Nozaki, K. Egashira, N. Hashimoto, Impact of monocyte chemoattractant protein-1 deficiency on cerebral aneurysm formation. Stroke 40, 942-951 (2009).

20. E. Ollikainen, R. Tulamo, J. Frösen, S. Lehti, P. Honkanen, J. Hernesniemi, M. Niemelä, P. T. Kovanen, Mast cells, neovascularization, and microhemorrhages are associated with saccular intracranial artery aneurysm wall remodeling. J. Neuropathol. Exp. Neurol. 73, 855-864 (2014).

21. D. Chyatte, G. Bruno, S. Desai, D. R. Todor, Inflammation and intracranial aneurysms. Neurosurgery 45, 1137-1146 (1999).

22. J. Frösen, A. Piippo, A. Paetau, M. Kangasniemi, M. Niemelä, J. Hernesniemi, J. Jääskeläinen, Remodeling of saccular cerebral artery aneurysm wall is associated with rupture: Histological analysis of 24 unruptured and 42 ruptured cases. Stroke 35, 2287-2293 (2004).

23. A. Terskikh, A. Fradkov, G. Ermakova, A. Zaraisky, P. Tan, A. V. Kajava, X. Zhao, S. Lukyanov, M. Matz, S. Kim, I. Weissman, P. Siebert, "Fluorescent timer": Protein that changes color with time. Science 290, 1585-1588 (2000).

24. M. Fukuda, T. Aoki, T. Manabe, A. Maekawa, T. Shirakawa, H. Kataoka, Y. Takagi, S. Miyamoto, S. Narumiya, Exacerbation of intracranial aneurysm and aortic dissection in hypertensive rat treated with the prostaglandin F-receptor antagonist AS604872. J. Pharmacol. Sci. 126, 230-242 (2014).

25. H. Hizaki, E. Segi, Y. Sugimoto, M. Hirose, T. Saji, F. Ushikubi, T. Matsuoka, Y. Noda, T. Tanaka, N. Yoshida, S. Narumiya, A. Ichikawa, Abortive expansion of the cumulus and impaired fertility in mice lacking the prostaglandin E receptor subtype EP2. Proc. Natl. Acad. Sci. U.S.A. 96, 10501-10506 (1999).

26. J. Y. Reuther, G. W. Reuther, D. Cortez, A. M. Pendergast, A. S. Baldwin Jr., A requirement for NF- $\kappa \mathrm{B}$ activation in $\mathrm{Bcr}-\mathrm{Abl}-$ mediated transformation. Genes Dev. 12, 968-981 (1998).

27. Y. Kanematsu, M. Kanematsu, C. Kurihara, Y. Tada, T.-L. Tsou, N. van Rooijen, M. T. Lawton, W. L. Young, E. I. Liang, Y. Nuki, T. Hashimoto, Critical roles of macrophages in the formation of intracranial aneurysm. Stroke 42, 173-178 (2011).

28. Y. Sugimoto, S. Narumiya, Prostaglandin E receptors. J. Biol. Chem. 282, 11613-11617 (2007).

29. M. S. Hayden, S. Ghosh, Shared principles in NF-KB signaling. Cell 132, 344-362 (2008).

30. S. Hou, H. Guan, R. P. Ricciardi, Phosphorylation of serine 337 of NF-kB p50 is critical for DNA binding. J. Biol. Chem. 278, 45994-45998 (2003).

31. T. Aoki, M. Fukuda, M. Nishimura, K. Nozaki, S. Narumiya, Critical role of TNF-alphaTNFR1 signaling in intracranial aneurysm formation. Acta Neuropathol. Commun. 2, 34 (2014).

32. R. M. Starke, D. M. S. Raper, D. Ding, N. Chalouhi, G. K. Owens, D. M. Hasan, R. Medel, A. S. Dumont, Tumor necrosis factor- $\alpha$ modulates cerebral aneurysm formation and rupture. Transl. Stroke Res. 5, 269-277 (2014). 
33. T. Grosser, S. Fries, G. A. FitzGerald, Biological basis for the cardiovascular consequences of COX-2 inhibition: Therapeutic challenges and opportunities. J. Clin. Invest. 116, 4-15 (2006).

34. A. Braeuning, S. Vetter, The nuclear factor $\kappa B$ inhibitor (E)-2-fluoro-4'-methoxystilbene inhibits firefly luciferase. Biosci. Rep. 32, 531-537 (2012).

35. J. J. Heynekamp, W. M. Weber, L. A. Hunsaker, A. M. Gonzales, R. A. Orlando, L. M. Deck, D. L. V. Jagt, Substituted trans-stilbenes, including analogues of the natural product resveratrol, inhibit the human tumor necrosis factor alpha-induced activation of transcription factor nuclear factor kappaB. J. Med. Chem. 49, 7182-7189 (2006).

36. J. G. Cui, Y. Y. Li, Y. Zhao, S. Bhattacharjee, W. J. Lukiw, Differential regulation of interleukin-1 receptor-associated kinase-1 (IRAK-1) and IRAK-2 by microRNA-146a and $\mathrm{NF}-\mathrm{KB}$ in stressed human astroglial cells and in Alzheimer disease. J. Biol. Chem. 285, 38951-38960 (2010).

37. J. Fan, F. T. Ishmael, X. Fang, A. Myers, C. Cheadle, S.-K. Huang, U. Atasoy, M. Gorospe, C. Stellato, Chemokine transcripts as targets of the RNA-binding protein HuR in human airway epithelium. J. Immunol. 186, 2482-2494 (2011).

38. D. Bai, Q. Gao, C. Li, L. Ge, Y. Gao, H. Wang, A conserved TGF $\beta 1 /$ HuR feedback circuit regulates the fibrogenic response in fibroblasts. Cell. Signal. 24, 1426-1432 (2012).

39. K. Paukku, M. Backlund, R. A. De Boer, N. Kalkkinen, K. K. Kontula, J. Y. Lehtonen, Regulation of AT1R expression through HuR by insulin. Nucleic Acids Res. 40, 5250-5261 (2012).

40. Y. Seko, H. Azmi, R. Fariss, J. A. Ragheb, Selective cytoplasmic translocation of HuR and site-specific binding to the interleukin-2 mRNA are not sufficient for CD28-mediated stabilization of the mRNA. J. Biol. Chem. 279, 33359-33367 (2004).

41. K. J. af Forselles, J. Root, T. Clarke, D. Davey, K. Aughton, K. Dack, N. Pullen, In vitro and in vivo characterization of PF-04418948, a novel, potent and selective prostaglandin EP2 receptor antagonist. Br. J. Pharmacol. 164, 1847-1856 (2011).

42. M. Sonobe, T. Yamazaki, M. Yonekura, H. Kikuchi, Small unruptured intracranial aneurysm verification study: SUAVe study, Japan. Stroke 41, 1969-1977 (2010).

43. D. Hasan, T. Hashimoto, D. Kung, R. L. Macdonald, H. R. Winn, D. Heistad, Upregulation of cyclooxygenase-2 (COX-2) and microsomal prostaglandin $E_{2}$ synthase-1 (mPGES-1) in wall of ruptured human cerebral aneurysms: Preliminary results. Stroke 43, 1964-1967 (2012).

44. B. A. Gross, P. M. Rosalind Lai, K. U. Frerichs, R. Du, Aspirin and aneurysmal subarachnoid hemorrhage. World Neurosurg. 82, 1127-1130 (2014).

45. D. M. Hasan, K. B. Mahaney, R. D. Brown Jr., I. Meissner, D. G. Piepgras, J. Huston, A. W. Capuano, J. C. Torner; International Study of Unruptured Intracranial Aneurysms Investigators, Aspirin as a promising agent for decreasing incidence of cerebral aneurysm rupture. Stroke 42, 3156-3162 (2011).

46. E. Garbe, S. H. Kreisel, S. Behr, Risk of subarachnoid hemorrhage and early case fatality associated with outpatient antithrombotic drug use. Stroke 44, 2422-2426 (2013).

47. R. A. Peña Silva, I. J. Mitchell, D. K. Kung, L. L. Pewe, M. F. Granja, J. T. Harty, F. M. Faraci, D. D. Heistad, D. M. Hasan, Paradoxical increase in mortality and rupture of intracranial aneurysms in microsomal prostaglandin E2 synthase type 1-deficient mice: Attenuation by aspirin. Neurosurgery 77, 613-620 (2015).

48. K. Kataoka, M. Taneda, T. Asai, A. Kinoshita, M. Ito, R. Kuroda, Structural fragility and inflammatory response of ruptured cerebral aneurysms. A comparative study between ruptured and unruptured cerebral aneurysms. Stroke 30, 1396-1401 (1999).

49. R. Tulamo, J. Frösen, S. Junnikkala, A. Paetau, J. Pitkäniemi, M. Kangasniemi, M. Niemelä, J. Jääskeläinen, E. Jokitalo, A. Karatas, J. Hernesniemi, S. Meri, Complement activation associates with saccular cerebral artery aneurysm wall degeneration and rupture. Neurosurgery 59, 1069-1076 (2006).

50. Y. Hui, E. Ricciotti, I. Crichton, Z. Yu, D. Wang, J. Stubbe, M. Wang, E. Puré, G. A. FitzGerald, Targeted deletions of cyclooxygenase-2 and atherogenesis in mice. Circulation 121, 2654-2660 (2010).

51. D. M. Hasan, K. B. Mahaney, V. A. Magnotta, D. K. Kung, M. T. Lawton, T. Hashimoto, H. R. Winn, D. Saloner, A. Martin, S. Gahramanov, E. Dósa, E. Neuwelt, W. L. Young, Macrophage imaging within human cerebral aneurysms wall using ferumoxytolenhanced MRI: A pilot study. Arterioscler. Thromb. Vasc. Biol. 32, 1032-1038 (2012).

52. Y. Ben-Neriah, M. Karin, Inflammation meets cancer, with NF- $\mathrm{KB}$ as the matchmaker. Nat. Immunol. 12, 715-723 (2011).
53. D. J. Rader, A. Daugherty, Translating molecular discoveries into new therapies for atherosclerosis. Nature 451, 904-913 (2008).

54. X. Ma, T. Aoki, T. Tsuruyama, S. Narumiya, Definition of prostaglandin $E_{2}-E P 2$ signals in the colon tumor microenvironment that amplify inflammation and tumor growth. Cancer Res. 75, 2822-2832 (2015).

55. R. Y. Cao, T. St. Amand, X. Li, S.-H. Yoon, C. P. Wang, H. Song, T. Maruyama, P. M. Brown, D. T. Zelt, C. D. Funk, Prostaglandin receptor EP4 in abdominal aortic aneurysms. Am. J. Pathol. 181, 313-321 (2012).

56. E. H. C. Tang, E. Shvartz, K. Shimizu, V. Z. Rocha, C. Zheng, D. Fukuda, G.-P. Shi, G. Sukhova, P. Libby, Deletion of EP4 on bone marrow-derived cells enhances inflammation and angiotensin II-induced abdominal aortic aneurysm formation. Arterioscler. Thromb. Vasc. Biol. 31, 261-269 (2011).

57. U. Yokoyama, R. Ishiwata, M.-H. Jin, Y. Kato, O. Suzuki, H. Jin, Y. Ichikawa, S. Kumagaya, Y. Katayama, T. Fujita, S. Okumura, M. Sato, Y. Sugimoto, H. Aoki, S. Suzuki, M. Masuda, S. Minamisawa, Y. Ishikawa, Inhibition of EP4 signaling attenuates aortic aneurysm formation. PLOS ONE 7, e36724 (2012).

58. J. Ando, K. Yamamoto, Flow detection and calcium signalling in vascular endothelial cells. Cardiovasc. Res. 99, 260-268 (2013).

59. E. Ollikainen, R. Tulamo, S. Lehti, M. Lee-Rueckert, J. Hernesniemi, M. Niemelä, S. Ylä-Herttuala, P. T. Kovanen, J. Frösen, Smooth muscle cell foam cell formation, apolipoproteins, and ABCA1 in intracranial aneurysms: Implications for lipid accumulation as a promoter of aneurysm wall rupture. J. Neuropathol. Exp. Neurol. 75, 689-699 (2016).

Acknowledgments: We thank Ono Pharmaceuticals for providing the EP2 agonist ONO-AE1-259 and R. Tulamo as well as J. Hernesniemi and M. Niemelä from the Neurosurgery Research Group and the Department of Neurosurgery at the Helsinki University Central Hospital for their help in the acquisition of tissue samples during microsurgical clipping of aneurysms. We also thank S. Morita from the Department of Biomedical Statistics and Bioinformatics at the Kyoto University Graduate School of Medicine for his help about the statistical analysis. We thank A. Mizutani, N. Asamoto, Y. Imai, and K. Mizutani for their technical assistance and also A. Washimi, K. Shiota, H. Kaimori, and T. Arai for their secretarial assistance. Funding: This work was supported by Core Research for Evolutional Science and Technology Program on Chronic Inflammation (to S.N.) from the Japan Science and Technology Agency (JST), by Coordination Fund from JST and Astellas Pharma Inc. (to S.N.), by collaborative grants to the Kyoto University from Ono Pharmaceuticals (to S.N.), and by a research grant from Finnish Medical Foundation and the Kuopio University Hospital (to J.F.). Author contributions: T.A., J.F., M.F., K.B., G.S., K.T., E.O., and J.L. performed the experiments. T.A., J.F., K.N., and S.N. designed the research. S.N. and J.F. provided funding. Competing interests: T.A. and S.N. are supported by Coordination Fund from JST and Astellas Pharma Inc. S.N. is a scientific advisor to Astellas Pharma Inc. The other authors declare that they do not have any competing interests. Data and materials availability: The Ptger2 flox mouse line, NF- $\mathrm{KB}$ reporter mouse line, and $/ \kappa B$ mutant transgenic mouse line were deposited in RIKEN, Japan with the accession numbers of CDB0939K, CDB0509T, and CDB0508T, respectively (www2.clst.riken.jp/ arg/mutant\%20mice\%20list.html, www2.clst.riken.jp/arg/TG\%20mutant\%20mice\%20list.html). Requests for these mice and established cell lines used will be accommodated under a material transfer agreement. Data from the microarray analysis (related to table S1 and data file S1) have been deposited in National Center for Biotechnology Information's Gene Expression Omnibus (GEO) and is accessible through GEO series accession number GSE93609 (www.ncbi.nlm.nih.gov/ geo/query/acc.cgi?acc=GSE93609).

Submitted 20 July 2016

Accepted 18 January 2017

Published 7 February 2017

10.1126/scisignal.aah6037

Citation: T. Aoki, J. Fròsen, M. Fukuda, K. Bando, G. Shioi, K. Tsuji, E. Ollikainen, K. Nozaki, J. Laakkonen, S. Narumiya, Prostaglandin $\mathrm{E}_{2}-\mathrm{EP} 2-\mathrm{NF}-\kappa \mathrm{B}$ signaling in macrophages as a potential therapeutic target for intracranial aneurysms. Sci. Signal. 10, eaah6037 (2017). 
Science Signaling

MAAAS
Prostaglandin $\mathrm{E}_{2}-\mathrm{EP2}-\mathrm{NF}-\mathrm{KB}$ signaling in macrophages as a potential therapeutic target for intracranial aneurysms

Tomohiro Aoki, Juhana Fr?sen, Miyuki Fukuda, Kana Bando, Go Shioi, Keiichi Tsuji, Eliisa Ollikainen, Kazuhiko Nozaki, Johanna Laakkonen and Shuh Narumiya (February 7, 2017)

Science Signaling 10 (465), . [doi: 10.1126/scisignal.aah6037]

The following resources related to this article are available online at http://stke.sciencemag.org. This information is current as of March 17, 2017.

Article Tools Visit the online version of this article to access the personalization and article tools:

http://stke.sciencemag.org/content/10/465/eah6037

Supplemental

Materials

Related Content

References

Permissions
"Supplementary Materials"

http://stke.sciencemag.org/content/suppl/2017/02/03/10.465.eaah6037.DC1

The editors suggest related resources on Science's sites:

http://stke.sciencemag.org/content/sigtrans/8/399/ra105.full

http://science.sciencemag.org/content/sci/332/6027/358.full

http://stm.sciencemag.org/content/scitransmed/5/183/183ra58.full

This article cites 59 articles, 29 of which you can access for free at: http://stke.sciencemag.org/content/10/465/eaah6037\#BIBL

Obtain information about reproducing this article: http://www.sciencemag.org/about/permissions.dtl

Science Signaling (ISSN 1937-9145) is published weekly, except the last December, by the American Association for the Advancement of Science, 1200 New York Avenue, NW, Washington, DC 20005. Copyright 2017 by the American Association for the Advancement of Science; all rights reserved. 